\title{
Scale-Invariant Model of Boltzmann Statistical Mechanics and Generalized Thermodynamics
}

\section{Modèle invariant d'échelles en mécanique statistique de Boltzmann et} thermodynamique généralisée

\author{
Siavash H. Sohrab ${ }^{1}$ \\ ${ }^{1}$ Northwestern University, Robert McCormick School of Engineering and Applied Science, Department of Mechanical \\ Engineering, 2145 Sheridan Road, Evanston, Illinois 60208, U.S.A., s-sohrab@northwestern.edu
}

\begin{abstract}
Some implications of a scale-invariant model of Boltzmann statistical mechanics to the laws of generalized thermodynamics are investigated. Through definition of stochastic Planck and Boltzmann universal constants, dimension of Kelvin absolute temperature $T$ (degrees kelvin) is identified as a length (meters) associated with Wien wavelength $T_{\beta}=\lambda_{\text {w } \beta}$ of particle thermal oscillations. Hence, thermodynamic temperature and atomic mass of the field $\mathbb{F}_{\beta}$ at scale $\beta$ provide internal measures of (extension, duration) of background space $\mathbb{S}_{\beta+1}=\mathbb{F}_{\beta}$ needed to define external space and time coordinates and atomic-mass-unit of $\mathbb{F}_{\beta+1}$. Introduction of invariant internal thermodynamic spacetime and Boltzmann factor are in harmony with modern concepts of quantum gravity as deterministic dissipative dynamic system [73]. The connections between de Pretto number 8338 and Joule-Mayer mechanical equivalent of heat $J_{\mathrm{c}}=4.169 \mathrm{~kJ} / \mathrm{kcal}$ and universal gas constant $R^{\mathrm{o}}=8338 \mathrm{~J} / \mathrm{kcal} . \mathrm{m}$ are identified leading to the modified mechanical equivalent of heat $J=2 J_{\mathrm{c}}=8338 \mathrm{~J} / \mathrm{kcal}$. It is shown that with work defined as Helmholtz free energy $W=F=U-T S$, Helmholtz decomposition of total thermal energy into free heat $U$ and latent heat $p V$ results in modified form of the first law of thermodynamics $Q=H=U-W=U+p V$. Finally, by application of Boltzmann combinatorics method, entropy of ideal gas is expressed in terms of the number of Heisenberg-Kramers virtual oscillators as $S=4 N \mathrm{k}$ in exact agreement with predicted entropy of black hole by Major and Setter [159].

KEYWORDS. Generalized thermodynamics, Entropy, Absolute temperature scale, Spacetime, Quantum nature of space and time, Quantum gravity, T.O.E.
\end{abstract}

\section{Introduction}

The laws of generalized thermodynamics and the underlying Boltzmann statistical mechanics appear to possess sufficient degree of universality to describe physical phenomena from extremely large cosmological scale to the minute scale of quantum optics. From cosmological observations, it is known that a galaxy such as the Milky Way (MW) that constitutes an "atom" of cosmic field is not at all visible at cosmological scales. However, as one approaches the MW, one first sees a small bright point appearing as a star, and only on a much closer approach one sees the enormous size of the entire MW galaxy extending about 100,000 light-years across. Similar descriptions apply to our solar system when viewed at galactic scales.

It is also known that a factor of approximately $10^{17}$ separates the scales of five major statistical fields in our universe starting with tachyon (graviton)-dynamics at exceedingly small Planck scale $10^{-35}$ $\mathrm{m}$, followed by electrodynamics $10^{-18} \mathrm{~m}$, hydrodynamics $10^{0} \mathrm{~m}$, astrophysics $10^{18} \mathrm{~m}$, and finally galactic-dynamics (cosmology) $10^{35} \mathrm{~m}$. Each statistical field has its characteristic "atomic" particle namely, graviton, electron, fluid-element, star, and galaxy. However, except their common similarities to turbulent phenomena, no global theoretical framework with sufficient degrees of freedom has yet been identified which allows for description of physical processes over diverse spectrum of spacetime. Clearly, systematic description of all motions of particles, both their momentum and energy, in such statistical fields require application of Boltzmann statistical mechanics. Since universality of Boltzmann statistical mechanics extends to the laws of generalized thermodynamics that govern 
macroscopic behavior of statistical fields at all scales, thermodynamics becomes a natural candidate for theory of everything (T.O.E.).

A scale-invariant model of Boltzmann statistical mechanics is schematically shown in Figure 1 based on similarities between stochastic quantum fields [1-17] on the one hand, and turbulent hydrodynamic fields [18-30] on the other hand. Recently, some of the implications of the model (Figure 1) to the fields of fluid mechanics [31,32], relativistic and classical thermodynamics [33,34], and quantum mechanics [35. 36] were examined. In addition, the gap between hydrodynamics and quantum mechanics was closed by derivation of invariant Schrödinger equation from invariant Bernoulli equation for incompressible potential flow [37].

In this study, implications of invariant Boltzmann statistical mechanics to the laws of generalized thermodynamics are further investigated. Since application of the model to relativistic [33] and classical [34] thermodynamics have been initiated, the study focuses on further clarification of concepts in generalized thermodynamics associated with hierarchies of embedded statistical fields. Also, in order to better reveal the nature of new paradigms and fundamental concepts such as temperature, heat, work, systems at thermodynamics equilibrium are considered that, once the "atom" of the field is defined, will be composed of a spectrum of atomic clusters that are stochastically stationary $[33,34,36]$. However, more complex models involving statistical fields at intermediate scales as well as non-equilibrium systems with non-local thermodynamics [38, 39, 40, 41, 42] are not addressed since the present study is focused on simple equilibrium systems.

In Section 2 the concept of statistical field $\mathbb{F}_{\beta}$ and background space $\mathbb{S}_{\beta+1}$ and their interconnections $\mathbb{S}_{\beta+1}=\mathbb{F}_{\beta}$ are discussed. It is shown that given the temperature and atomic mass of $\mathbb{F}_{\beta}$ is sufficient to define internal spacetime of the background "space" $\mathbb{S}_{\beta+1}$ hence external independent space and time coordinates as well as the atomic-mass-unit of $\mathbb{F}_{\beta+1}$ (Figure 1). Next, in Sections 3-6, new perspectives concerning the zeroth, first, second, and third law of generalized thermodynamics are presented to compliment the earlier investigations [33-37].

In the course of the present study, it is necessary to discuss possible implications of the model to the diverse fields of cosmology, astrophysics, hydrodynamics, molecular dynamics, electrodynamics, and tachyon(graviton)-dynamics. Clearly, in order to achieve a universal perspective of the physical laws over a wide range of spacetime, considerations of at least the fundamentals of different branches of physical sciences become necessary. Obviously, the discussion of all these various fields of scientific disciplines does not imply the proficiency of the author in any one of them.

The question may be raised as to why such comprehensive global modification of our physical concepts pertaining to the laws of nature are needed if these laws have been derived from the systematic application of scientific method during the evolution of the history of science. It is noted however, that although the tree of knowledge has grown to such magnificent proportions, bearing such variety of useful fruits, the very roots of this tree were formed at a time of relative scientific ignorance. As a result, our understanding of some of the most fundamental scientific concepts such as space, time, mass, temperature, etc. lack exact scientific precision. Of course, the great stability of the tree of knowledge throughout history can be attributed to the empirical nature of many of its roots. In fact, it is the strict requirement of consistency with the empirical observations that distinguishes scientific method from philosophical speculations. Therefore, the new paradigms must not only conform to all empirical observations, but also provide for a more unitary approach to their descriptions, resort to minimum number of postulates while making new predictions and extending the horizon for future scientific developments. 


\section{Boltzmann and Scale-Invariant Model of Statistical Mechanics}

Following classical methods [19, 43-47], invariant density $\rho_{\beta}$, and velocity of atom $\mathbf{u}_{\beta}$, element $\mathbf{v}_{\beta}$, and system $\mathbf{w}_{\beta}$ (Figure 1) at the scale $\beta$ are given as [36]

$$
\begin{array}{lll}
\rho_{\beta}=\mathrm{n}_{\beta} \mathrm{m}_{\beta}=\mathrm{m}_{\beta} \int \mathrm{f}_{\beta} \mathrm{du} \mathbf{u}_{\beta} & , & \mathbf{u}_{\beta}=\mathbf{v}_{\mathrm{mp} \beta-1} \\
\mathbf{v}_{\beta}=\rho_{\beta}^{-1} \mathrm{~m}_{\beta} \int \mathbf{u}_{\beta} \mathrm{f}_{\beta} \mathrm{d} \mathbf{u}_{\beta} & , & \mathbf{w}_{\beta}=\mathbf{v}_{\mathrm{mp} \beta+1}
\end{array}
$$

leading to peculiar and diffusion velocities

$$
\mathbf{V}_{\beta}^{\prime}=\mathbf{u}_{\beta}-\mathbf{v}_{\beta} \quad, \quad \mathbf{V}_{\beta}=\mathbf{v}_{\beta}-\mathbf{w}_{\beta} \quad, \quad \mathbf{V}_{\beta}=\mathbf{V}_{\beta+1}^{\prime}
$$

The statistical fields of equilibrium galactic-, planetary-, hydro-system-, fluid element-, eddy-, cluster-, molecular-, atomic-, subatomic-, kromo-, and tachyon-dynamics at the scale $\beta=\mathrm{g}, \mathrm{p}, \mathrm{h}, \mathrm{f}, \mathrm{e}, \mathrm{c}$, $\mathrm{m}, \mathrm{a}, \mathrm{s}, \mathrm{k}$, and $\mathrm{t}$ and the corresponding nonequilibrium laminar flow fields are schematically shown on the left- and right-hand sides of Figure 1. Each statistical field is governed by distribution function $\mathrm{f}_{\beta}\left(\mathbf{x}_{\beta}, \mathbf{u}_{\beta}, \mathrm{t}_{\beta}\right)$ and is constructed from its "atom" viewed as point-mass and a part of the background field of lower scale. Next, ensembles of "atoms" form finite-sized composite entities called "elements" of the field. Finally, collection of all "elements" is defined as the statistical "system" at that particular scale. The most-probable element of scale $\beta$ defines the "atom" of the next higher scale $\beta+1$ such that.

$\mathbf{v}_{\mathrm{mp} \beta}=\mathbf{u}_{\beta+1}$. Also, the most-probable element of scale $\beta$ defines the system of the next lower scale $\beta-1, \mathbf{v}_{\mathrm{mp} \beta}=\mathbf{w}_{\beta-1}$ thus resulting in hierarchy of statistical fields schematically shown in Figure 1. As was discussed in [36], in view of the estimated scale factor of approximately $10^{-26}$ between mass of graviton (tachyon) versus photon, it is reasonable to anticipate another statistical field called equilibrium-neutrino-dynamics END $\beta=\mathrm{n}$, not shown in Figure 1, at an intermediate scale separating EKD $\beta=\mathrm{k}$ from $\operatorname{ETD} \beta=\mathrm{t}$ field.

Beginning with the scale of laminar molecular dynamics LMD, molecules, clusters of molecules, and cluster of clusters of molecules (eddy) are "atom", "element", and "system" of LMD field with the velocities $\left(\mathbf{u}_{\mathrm{m}}, \mathbf{v}_{\mathrm{m}}, \mathbf{w}_{\mathrm{m}}\right)$. Similarly, the fields of laminar cluster-dynamics LCD and eddy-dynamics LED will have the velocities $\left(\mathbf{u}_{\mathrm{c}}, \mathbf{v}_{\mathrm{c}}, \mathbf{w}_{\mathrm{c}}\right)$ and $\left(\mathbf{u}_{\mathrm{e}}, \mathbf{v}_{\mathrm{e}}, \mathbf{w}_{\mathrm{e}}\right)$ by Equations (1)-(2).

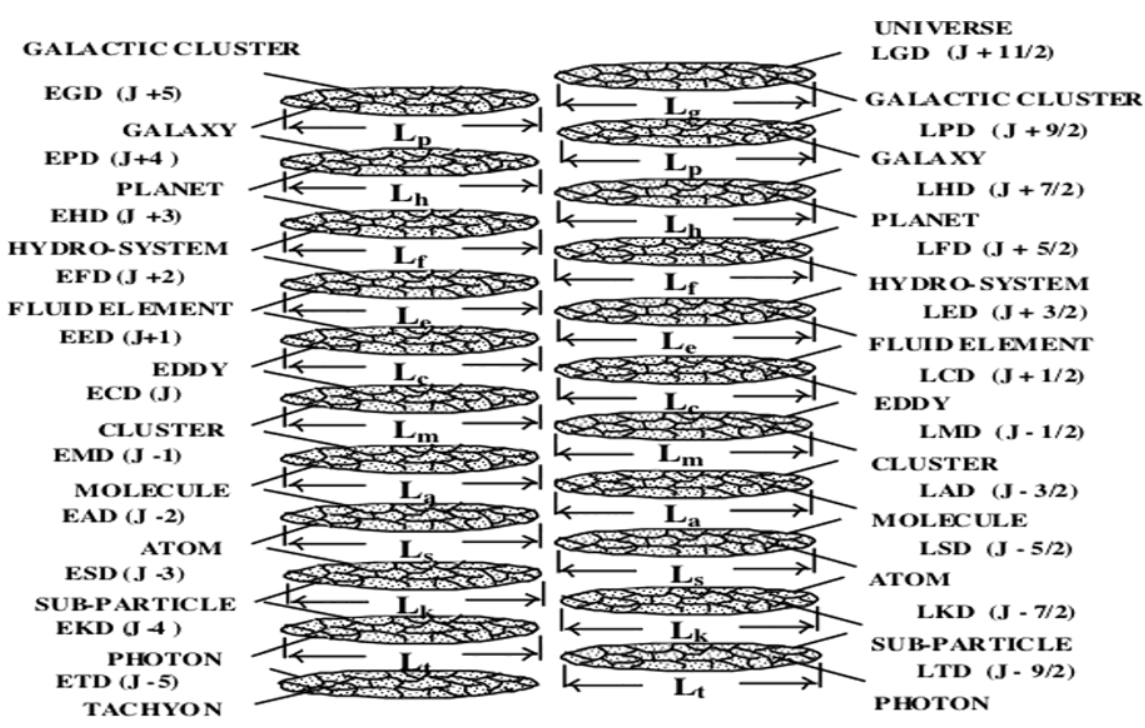

Figure 1. Scale-invariant model of statistical mechanics. Equilibrium- $\beta$-dynamics of the left-hand side and nonequilibrium laminar- $\beta$-dynamics on the right-hand side for scales $\beta=g, p, h, t, e, c, m, a, s, k$, and $t$ as defined in Sec. 2. Lengths of (system, element, atom) are $\left(\mathrm{L}_{\beta}, \lambda_{\beta}, \ell_{\beta}\right)$ and $\lambda_{\beta}$ is the mean-free-path [36] 
Typical (atom, element, system) lengths at (EED, ECD, EMD) scales are

$$
\begin{aligned}
& \operatorname{EED} \quad\left(\ell_{\mathrm{e}}, \lambda_{\mathrm{e}}, \mathrm{L}_{\mathrm{e}}\right)=\left(10^{-5}, 10^{-3}, 10^{-1}\right) \mathrm{m} \\
& \operatorname{ECD} \quad\left(\ell_{\mathrm{c}}, \lambda_{\mathrm{c}}, \mathrm{L}_{\mathrm{c}}\right)=\left(10^{-7}, 10^{-5}, 10^{-3}\right) \mathrm{m} \\
& \operatorname{EMD} \quad\left(\ell_{\mathrm{m}}, \lambda_{\mathrm{m}}, \mathrm{L}_{\mathrm{m}}\right)=\left(10^{-9}, 10^{-7}, 10^{-5}\right) \mathrm{m}
\end{aligned}
$$

The relative system sizes of these statistical fields are schematically shown in Figure 2.

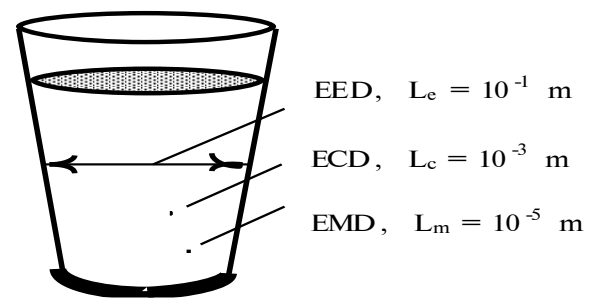

Figure 2. Lengths of (system, element, atom) are $\left(\mathrm{L}_{\beta}, \lambda_{\beta}, \ell_{\beta}\right)$ and $\lambda_{\beta}$ is the mean-free-path [36]

Applying the same $\left(\ell_{\beta}, \lambda_{\beta}, \mathrm{L}_{\beta}\right)$ as in Equation (4) and relation $\ell_{\beta}=\lambda_{\beta-1}=\mathrm{L}_{\beta-2}$ between scales to Figure 1 results in the hierarchy of overlapping statistical fields schematically shown in Figure 3. In Figure 3, starting from Planck length $\left(\hbar \mathrm{G} / \mathrm{c}^{3}\right)^{1 / 2} \approx 10^{-35} \mathrm{~m}$ where $\mathrm{G}$ is the gravitational constant, seven generations of statistical fields or a factor of about $10^{17}$ separates the five major statistical fields of tachyon(graviton)-dynamic $10^{-35}$, electro-dynamic $10^{-18}$, hydrodynamics $10^{0}$, astrophysics $10^{18}$, and finally galactic-dynamics or cosmology $10^{35} \mathrm{~m}$.

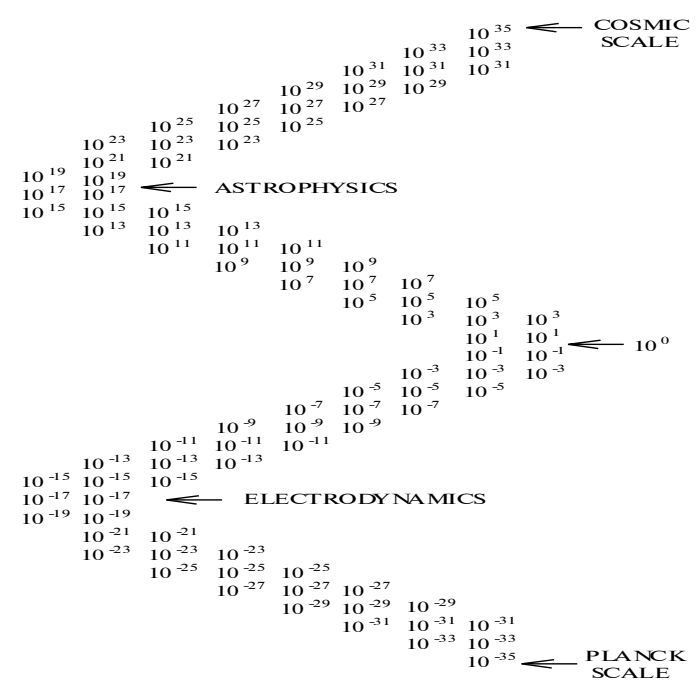

Figure 3. Hierarchy of statistical fields with chracteristic (atom, element, system)lenngth scales

$$
\left(\ell_{\beta}, \lambda_{\beta}, \mathrm{L}_{\beta}\right) \text { from cosmic to Planck scales [36] }
$$

Since invariant Schrödinger equation was derived from invariant Bernoulli equation [37], all statistical fields in Figure 1 are governed by quantum mechanics. Due to the absence of any physical or mathematical reasons to the contrary, the hierarchy in Figure 3 could continue to larger (many universe) and smaller (sub-tachyonic) scales ad infinitum. Also, notwithstanding the often-quoted statement: "God does not play dice" by Einstein, according to Figure 3, the question is not whether Almighty plays dice but rather, "How does God play dice?" [48]. Interestingly, with a transparent material such as Lucite (Plexiglass), one can construct a simple system of finite embedded dice as schematically shown in Figure 4. 


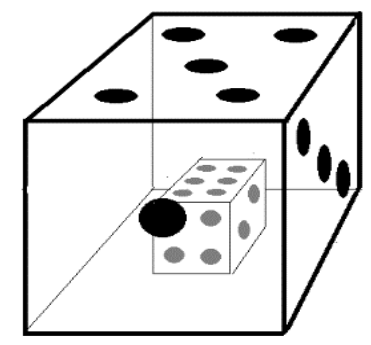

Figure 4. Hierarchy in Figures 1 and 3 like embedded dice show that chance is the essence of all existence from cosmic to sub-photonic scales

Also, universal nature of Schrödinger equation [37] leads to universal probabilistic nature of the laws of nature in view of probabilistic interpretation of wave function [49]. This not only ensures stochastic law of justice in nature but also saves the grace because in an absolutely deterministic Laplacian world, an absolutely benevolent God could not justify the presence of any evil, whereas in a probabilistic world all evil could be attributed to chance.

The universality of invariant Schrödinger equation suggests that the laws of nature over the entire spectrum of statistical fields shown in Figures 1 and 3 are governed by quantum mechanics and hence are probabilistic as is to be expected. Of course, this does not mean that deterministic systems of classical mechanics do not play their central role in modelling physical phenomena. The new paradigm of physical foundation of quantum mechanics discussed in [37] is in harmony with the perceptions of Heisenberg [50] in regards to the domain of applicability of classical versus quantum mechanics,

"We no longer say "Newtonian mechanics is false and must be replaced by quantum mechanics, which is correct." Instead, we adopt the formula "Classical mechanics is a consistent self-enclosed scientific theory. It is strictly correct description of nature wherever its concepts can be applied"

Due to its statistical foundation, quantum mechanics concerns behavior of Heisenberg-Kramers [51] virtual oscillators or de Broglie [3] wave packets in statistical fields such as cosmology, astrophysics, hydrodynamics, molecular dynamics, electrodynamics or optics [37]. Classical Newtonian mechanics on the other hand is mainly concerned with motion of a few bodies such as the classical Poincare [52] three-body-problem of earth-moon-sun that resulted in his discovery of chaos theory.

When particles are assumed to act as composite bosons [53, 54] made of "cooper pairs" of atoms and follow corrected Boltzmann statistics [55,56], velocity, energy, and speed of particles at the state of thermodynamic equilibrium will be respectively governed by invariant Gaussian (Maxwell), Planck, and Maxwell-Boltzmann distribution functions [35,36]

$$
\begin{aligned}
& \mathrm{f}_{\beta}\left(\mathrm{v}_{\beta}\right)=\left(\frac{\mathrm{m}_{\beta}}{2 \pi \mathrm{k} T_{\beta}}\right)^{3 / 2} \mathrm{e}^{-\mathrm{m}_{\beta} \mathrm{u}_{\beta}^{2} / 2 \mathrm{k} T_{\beta}} \\
& \frac{\varepsilon_{\beta} d N_{\beta}}{V}=\frac{8 \pi \mathrm{h}}{\mathrm{u}_{\beta}^{3}} \frac{v_{\beta}^{3}}{\mathrm{e}^{\mathrm{h} \mathrm{v}_{\beta} / k T}-1} \mathrm{~d} v_{\beta} \\
& \frac{d N_{\mathrm{u} \beta}}{N}=4 \pi\left(\frac{\mathrm{m}_{\beta}}{2 \pi \mathrm{k} T_{\beta}}\right)^{3 / 2} \mathrm{u}_{\beta}^{2} \mathrm{e}^{-\mathrm{m}_{\beta} \mathrm{u}_{\beta}^{2} / 2 \mathrm{k} T_{\beta}} \mathrm{du}_{\beta}
\end{aligned}
$$

For example, the invariant Maxwell-Boltzmann distribution in Equation (5c) is applied to calculate the speed distributions at three consecutive scales of equilibrium (cluster, molecular, atomic) dynamics (ECD, EMD, EAD) at a constant temperature $T=300 \mathrm{~m}$ as shown in Figure 5. 


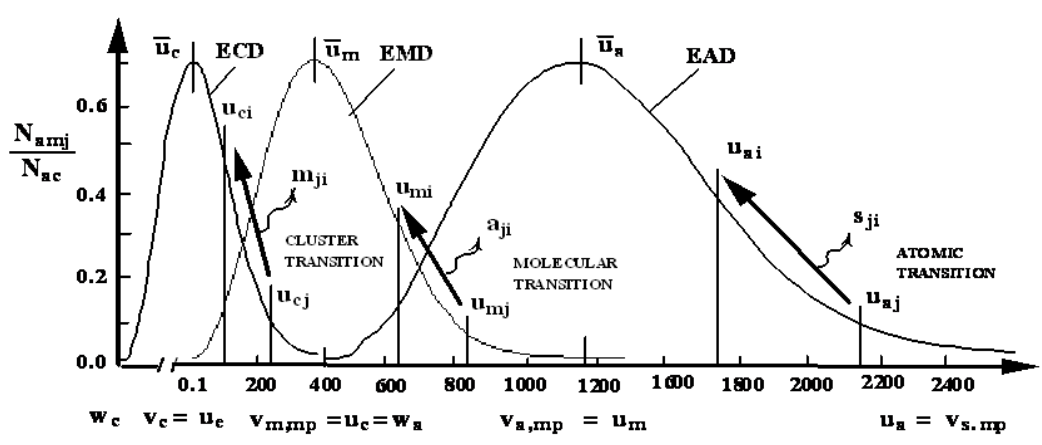

Figure 5. Maxwell-Boltzmann distribution of (ECD, EMD, EAD) at $T=300 \mathrm{~m}$ [36]

Starting with an atom $\mathbf{u}_{\beta}$ that is stabilized by an external pressure known as Poincare stress [35], spectrum of atomic clusters with velocity $\mathbf{v}_{\mathrm{j \beta}}$ form an equilibrium statitical field when absolute thermodynamic temeprature defined as kientic energy of the most probable cluster $\mathrm{kT}_{\beta}=\mathrm{m}_{\beta} \mathrm{v}_{\mathrm{mp} \beta}^{2}$ becomes stochastically stationary (Figure 5).

As discussed in [36], the conventional field of fluid dynamics does not correspond to laminar molecular dynamics LMD $\beta=\mathrm{m}$ but rather to the next higher scale of laminar cluster-dynamics LCD $\beta=c$ becuase at EMD scale, the root-mean-square speed of molecules is the speed of sound [37,57] that does not correspond to local velocity in hydrodynamics. Hence, in stationary fluid at ECD scale, Maxwell-Boltzmann distribution function governs stochastically stationary cluster sizes. Random motion of clusters accounts for the Brownian motion of small suspensions that is known to be a nondissipative stationary phenomenon [35]. Therefore, Maxwell demon paradox discussed by Poincaré [58] is resolved since Brownian particles will be in equilibrium with clusters in ECD field (Figures 1 and 2) that are undergoing Brownian motions themselve. Such a scale-invariant model of "Brownian motions" is in accordance with perceptions of Gouy [59] and harmonious with classical theories of Brownian motions [60-70].

Although atoms are under constant transitions between different clusters, also called energy-levels as shown in Figure 5, clusters remain stochastically stationary due to the principle of detailed balance [36]. For example, in isotropic stationary homogeneous turbulence corresponding to EED, transition of a cluster from a small fast-oscillating "eddy-j" (high energy-level-j) to a large slowly-oscillating "eddyi" (low energy-level-i) results in emission of a "sub-particle" that is a molecule to carry away the excess energy in harmony with Bohr [71] frequency formula $\Delta \varepsilon_{\mathrm{j} i \beta}=\mathrm{h}\left(v_{\mathrm{j} \beta}-v_{\mathrm{i} \beta}\right)$ as schematically shown in Figure 6a.

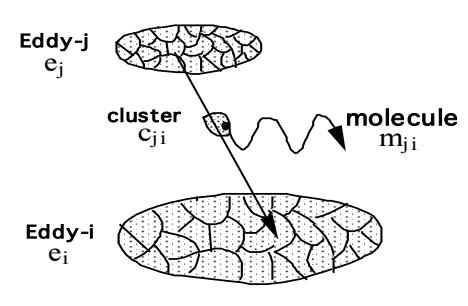

(a)

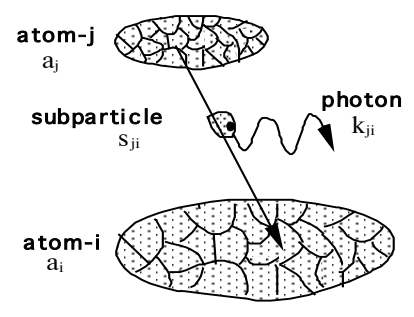

(b)

Figure 6. (a) Transition of cluster from eddy-j to eddy-i leading to emission of molecule. (b) Transitions of Electron from atom-j to atom-i leading to emission of photon [37]

Similarly, in equilibrium-electro-dynamics ESD $\beta=$ s, i.e. stochastic electrodynamics (SED), transition of an electron from a small fast-oscillating "atom-j" (high energy-level-j) to a large slow- 
oscillating "atom-i" (low energy-level-i) leads to emission of a sub-particle photon $\gamma_{\mathrm{ji}}$ as shown in Figure 6b. According to the model in Figure 6, any particle of cluster-j could go through any trajectory to cluster-i emitting energy $\Delta \varepsilon_{\mathrm{j} \beta}=\mathrm{h}\left(v_{\mathrm{j} \beta}-v_{\mathrm{i} \beta}\right)$ with the same final state. By Equation (90) of Section 5, Boltzmann factor governs transition probabilities hence particle trajectories between different clusters (energy levels) thus accounting for the success of Feynman integral over all paths.

The new paradigm of physical foundation of quantum mechanics and transitions between particle clusters, de Broglie wave-packets (Figure 6), is in harmony with quantum transitions between different "cells" in recent cellular automaton interpretation of quantum mechanics [72]. The central role of equilibrium thermodynamics in quantum theory of gravity has been emphasized by 't Hooft [73]

"Models describing only a small number of quantum states, such as all the above, do not show very clearly the most salient difficulty encountered when one attempts to construct realistic models. This is the fact that our universe is known to be thermodynamically stable. A system in thrermodynamic equilibrium is governed by Boltzmann factor $e^{-\beta E_{i}}$, where $\beta$ is the inverse temperature."

In Figure 6, atomic transitions between clusters (energy-levels) $(i \rightarrow j),(j \rightarrow k)$ are governed by transition enthalpies $H_{\mathrm{ij}}=4 k T\left(N_{\mathrm{i}}-N_{\mathrm{j}}\right), \quad H_{\mathrm{jk}}=4 k T\left(N_{\mathrm{j}}-N_{\mathrm{k}}\right)$ that occur in Boltzmann factors $\mathrm{p}_{\mathrm{ij}}=e^{-H_{\mathrm{ij}} / \mathrm{k} T}$ and $\mathrm{p}_{\mathrm{jk}}=e^{-H_{\mathrm{j} \mathrm{k}} / \mathrm{k} T}$ by Equation (90), such that $\mathrm{p}_{\mathrm{ij}} \cdot \mathrm{p}_{\mathrm{jk}}=\mathrm{p}_{\mathrm{ik}}$ in harmony with Heisenberg [74] matrix mechanics. At the scale of Casimir [75] vacuum or physical space $\beta=k$ (Figure 1), since $\mathrm{p}_{\mathrm{ij}} \neq \mathrm{p}_{\mathrm{ji}}$ follows Heisenberg [51,74] matrix mechanics, physical space will be governed by noncommutative geometry discussed by Connes [76].

According to the invariant model of Boltzmann statistical mechanics (Figure 1), the statistical field $\mathbb{F}_{\beta}$ at scale $\beta$ resides within and is in thermodynamics equilibrium $T_{\beta}=T_{\beta-1}$ with the background "space" $\mathbb{S}_{\beta}$ that is identified as the statsitical field at the next lower scale $\mathbb{S}_{\beta}=\mathbb{F}_{\beta-1}$. For example, given $T_{\beta-1}$ hence internal spacetime of $\mathbb{F}_{\beta-1}$ in astrophysics $\beta-1=\mathrm{s}$, one can define external space and time coordimates $\left(\mathrm{x}_{\beta}, \mathrm{t}_{\beta}\right)=\left(\mathrm{N}_{\mathrm{x}} \lambda_{\mathrm{w \beta -1}-1}, \mathrm{~N}_{\mathrm{t}} \tau_{\mathrm{w \beta}-1}\right)$ at scale $\beta=\mathrm{g}$ of cosmology. Hence, every point of background "space" $\mathbb{S}_{\beta}=\mathbb{F}_{\beta-1}$ in cosmology is occupied by either an atom i.e., a galaxy, or else by Casimir [75] vacuum as discussed in [77]. Also, since in classical physics, atomic masses are defined in terms of ати that is identified as electromagnetic mass of photon in Equation (17) of the following Section, given $\mathbb{F}_{\beta-1}\left(T_{\beta-1}, \mathrm{~m}_{\beta-1}\right)$, atomic-mass-unit of scale $\beta$ is defined as the electromagnetic mass of atom of lower scale $\beta-1$

$$
a m u_{\beta}=3 \mathrm{kT}_{\beta-1}=3 \mathrm{~m}_{\beta-1} \mathrm{v}_{\mathrm{w} \beta-1}^{2}=\mathrm{m}_{\beta-1} \mathrm{v}_{\mathrm{r} \beta-1}^{2}=\mathrm{m}_{\beta-1} \mathrm{c}_{\mathrm{s} \beta-1}^{2}=\hat{\mathrm{u}}_{\beta-1}
$$

where $c_{s \beta-1}$ is the root-mean-square speed or speed of "sound" [37, 57]. In general, mass at scale $\beta$ is defined as $M_{\beta}=N_{\beta}\left(a m u_{\beta}\right)$. Thus, $\mathbb{F}_{\beta-1}\left(T_{\beta-1}, \mathrm{~m}_{\beta-1}\right)$ represents the "space" $\mathbb{S}_{\beta}=\mathbb{F}_{\beta-1}$ wihtin which one can define (length, time, mass) $=\left(\mathrm{x}_{\beta}, \mathrm{t}_{\beta}, \mathrm{M}_{\beta}\right)$, for example $(\mathrm{m}, \mathrm{s}, \mathrm{kg})$, of a new statsitical field $\mathbb{F}_{\beta}\left(T_{\beta}, \mathrm{m}_{\beta}\right)$ leading to the hierarchy of embedded fields

$$
\mathbb{F}_{\beta}\left[\mathbb{F}_{\beta-1}\left(T_{\beta-1}, \mathrm{~m}_{\beta-1}\right)\left[\mathbb{F}_{\beta-2}\left(T_{\beta-2}, \mathrm{~m}_{\beta-2}\right)[\ldots]\right]\right.
$$


As a metaphor, the three independent variables (space, time, mass) $=\left(\mathrm{x}_{\beta}, \mathrm{t}_{\beta}, \mathrm{M}_{\beta}\right)$, like the three primary colors (red, yellow, blue), are all that is needed to paint the entire tapestry of creation from cosmic to photonic scales (Figures 1 and 3).

The universal nature of quantum fields in Equation (7) is in harmony with perceptions of Rovelli as described in Figure 7.8 of his book [78] stating that covariant quantum fields are all that is needed to define (space, time, particle) in theory of quantum gravity. The role of thermodynamics as one of the three primary ingrediants of quantum gravity as universal theory of nature has been emphasized by Rovelli [78]

"All of this, I believe, indicates that in order to grasp the basic grammar of the world, we need to merge three basic ingredients, not just two: not just general relativity and quantum mechanics, but also the theory of heat, that is, statistical mechanics and thermodynamics, which we can also describe as "information theory". But the thermodynamics of general relativity - that is to say, the statistical mechanics of quanta of space-is as yet only in its early infancy. Everything is still confused, and there is a very great deal that remains to be understood."

Invariant model of Boltzmann statistical mechanics (Figures 1,3) and the corresponding concepts regarding relativistic and classical thermodynamics [33,34] and quantum mechanics [35,36,37] are harmonious with modern theoretical concepts of quantum gravity [73,76,78,79,80,81,82] As discussed above, when space or Casimir [75] vacuum is identified as a compressible tachyon fluid, a new paradigm for physical foundation of quantum gravity [83, 84] becomes possible. Because of the definition of internal atomic time [77], quantum theory of gravity will have wave function $\Psi_{\beta}$ that instead of Wheeler-DeWitt equation [81,82] satisfies the invariant Schrödinger equation [37]. The resurrection of time in Wheeler-DeWitt equation becomes possible because the new "atomic" time arises from internal degrees of freedom associated with thermodynamic temperature of cosmic field [77].

Given $\mathbb{F}_{\beta}\left(T_{\beta}, \mathrm{m}_{\beta}\right)$ and hence independent space and time coordinates as well as atomic mass, following the classical methods [19, 43-47], the scale-invariant forms of mass, thermal energy, linear and angular momentum conservation equations for reactive fields at scale $\beta$ are given as [36]

$$
\begin{aligned}
& \frac{\partial \rho_{\mathrm{i} \beta}}{\partial t_{\beta}}+\nabla \cdot\left(\rho_{\mathrm{i} \beta} \mathbf{v}_{\beta}\right)=\mathfrak{R}_{\mathrm{i} \beta} \\
& \frac{\partial \varepsilon_{\mathrm{i} \beta}}{\partial t_{\beta}}+\nabla \cdot\left(\varepsilon_{\mathrm{i} \beta} \mathbf{v}_{\beta}\right)=0 \\
& \frac{\partial \mathbf{p}_{\mathrm{i} \beta}}{\partial t_{\beta}}+\nabla \cdot\left(\mathbf{p}_{\mathrm{i} \beta} \mathbf{v}_{\beta}\right)=-\nabla \cdot \mathbf{P}_{\mathrm{ij} \beta} \\
& \frac{\partial \pi_{\mathrm{i} \beta}}{\partial t_{\beta}}+\nabla \cdot\left(\boldsymbol{\pi}_{\mathrm{i} \beta} \mathbf{v}_{\beta}\right)=\rho_{\mathrm{i} \beta} \boldsymbol{\omega}_{\beta} \cdot \nabla \mathbf{v}_{\beta}
\end{aligned}
$$

with volumetric density of thermal energy $\varepsilon_{\mathrm{i} \beta}=\rho_{\mathrm{i} \beta} \tilde{h}_{\mathrm{i} \beta}$, linear momentum $\mathbf{p}_{\mathrm{k}}=\rho_{\mathrm{i} \beta} \mathbf{v}_{\mathrm{i} \beta}$, and angular momentum $\pi_{\mathrm{i} \beta}=\rho_{\mathrm{i} \beta} \omega_{\mathrm{i} \beta}\left(\right.$ since $\left.\mathbf{r}_{\mathrm{a} \beta-1}=1\right)$. Also, $\mathfrak{R}_{\mathrm{i} \beta}$ is the chemical reaction rate and $\tilde{h}_{\mathrm{i} \beta}=\hat{h}_{\mathrm{i} \beta} / \mathrm{m}_{\beta}$ is absolute enthalpy [36]. It is noted that the time coordinates in Equations (8-11) also have a scale subscript $\beta$ [77]. 


\section{Kelvin Absolute Temperature Scale and Zeroth Law of Thermodynamics}

As discussed in [33,34], a fundamental shift of paradigm concerns the change of dimension of Kelvin absolute thermodynamic temperature $T$ from (degrees kelvin) to length (meter). Clearly, changing the dimension of temperature will have a global impact on all branches of natural sciences. Such a fundamental change of scientific paradigm is mandated by the new definitions of stochastic Planck $\mathrm{h}$ and Boltzmann $\mathrm{k}$ universal constants [33,34]. Finally, because temperature is a measured property, its dimension must be ultimately based on the theory that defines it as emphasized by Planck [85].

"Every measurement first acquires its meaning for physical science through the significance which a theory gives it"

Because dimensions of all other quantities in physics are based on three fundamental dimensions namely (length, time, mass) that define $\mathbb{F}_{\beta}\left(\mathrm{x}_{\beta}, \mathrm{t}_{\beta}, \mathrm{M}_{\beta}\right)$, the name degrees kelvin does not reveal the true dimension of absolute temperature since "degree kelvin" is not a fundamental physical dimension ( $\mathrm{m}$, $\mathrm{s}, \mathrm{kg}$ ). Also, in view of atomic energy $\mathrm{k} T_{\beta}=\hat{\varepsilon}_{\beta}$, dimensions of both $T$ and Boltzmann constant $\mathrm{k}$ should be simultaneously modified.

Since the mean particle velocity must vanish $\left\langle\mathbf{u}_{\mathrm{k}}\right\rangle=0$ at thermodynamic equilibrium, particle energy $\varepsilon_{\beta}=\mathrm{m}_{\beta}<\mathrm{u}_{\beta}^{2}>=\overline{\mathrm{p}}_{\beta}<\lambda_{\beta}^{2}>^{1 / 2}<v_{\beta}^{2}>^{1 / 2}$ can be expressed in terms of either frequency $\varepsilon_{\beta}=\mathrm{h}_{\beta}<v_{\beta}^{2}>^{1 / 2}$ or wavelength $\varepsilon_{\beta}=\mathrm{k}_{\beta}<\lambda_{\beta}^{2}>^{1 / 2}$ when stochastic (Planck, Boltzmann) factors are defined as $\left(\mathrm{h}_{\beta}=\overline{\mathrm{p}}_{\beta}<\lambda_{\beta}^{2}>^{1 / 2}, \mathrm{k}_{\beta}=\overline{\mathrm{p}}_{\beta}<\mathrm{v}_{\beta}^{2}>^{1 / 2}\right)$ and $\overline{\mathrm{p}}_{\beta}=\mathrm{m}_{\beta}<\mathrm{u}_{\beta}^{2}>^{1 / 2}$ is the root-mean-square momentum of particle $[33,34]$. As discussed in Section 4 , particles are considered to have harmonic translational motions in two directions such that $\varepsilon_{\mathrm{t} \beta}=\mathrm{m}_{\beta}<\mathrm{u}_{\mathrm{x}+\beta}^{2}>/ 2+\mathrm{m}_{\beta}<\mathrm{u}_{\mathrm{x}-\beta}^{2}>/ 2=\mathrm{m}_{\beta}<\mathrm{u}_{\mathrm{x}+\beta}^{2}>$. At EKD scale $\beta=\mathrm{k}$ (Figure 1), the system is identified as physical space or Casimir vacuum [75] that may be also called Aristotle fifth element, de Broglie hidden thermostat [3], or Dirac stochastic ether [86]. Naturally, Planck and Boltzmann factors are identified as Planck and Boltzmann universal constants associated with spatial $<\lambda_{\beta}^{2}>^{1 / 2}$ and temporal $<v_{\beta}^{2}>^{1 / 2}$ aspects of Casimir [75] vacuum fluctuations [33,34]

$$
\begin{aligned}
& \mathrm{h}=\mathrm{h}_{\mathrm{k}}=\overline{\mathrm{p}}_{\mathrm{k}}\left\langle\lambda_{\mathrm{k}}^{2}\right\rangle^{1 / 2}=\mathrm{m}_{\mathrm{k}} \mathrm{c} \lambda_{\text {rmsk }}=\mathrm{m}_{\mathrm{k}} \mathrm{c} \lambda_{\mathrm{rk}}=6.626 \times 10^{-34}[\mathrm{~J}-\mathrm{s}] \\
& \mathrm{k}=\mathrm{k}_{\mathrm{k}}=\overline{\mathrm{p}}_{\mathrm{k}}\left\langle v_{\mathrm{k}}^{2}\right\rangle^{1 / 2}=\mathrm{m}_{\mathrm{k}} \mathrm{c} v_{\text {rmsk }}=\mathrm{m}_{\mathrm{k}} \mathrm{c} v_{\mathrm{rk}}=1.381 \times 10^{-23}=[\mathrm{J} / \mathrm{m}]
\end{aligned}
$$

By definition $\overline{\mathrm{p}}_{\mathrm{k}}=\overline{\mathrm{p}}_{\mathrm{k}}=\mathrm{mv}_{\mathrm{rk}}=\mathrm{mc}$, Planck and Boltzmann constants $(\mathrm{h}, \mathrm{k})$ are respectively defined in terms of root-mean-square wavelength and frequency $\left(\lambda_{\mathrm{rmsk}}, v_{\mathrm{rmsk}}\right)=\left(\lambda_{\mathrm{rk}}, v_{\mathrm{rk}}\right)$ where $\lambda_{\mathrm{rk}} v_{\mathrm{rk}}=\mathrm{c}$ and $\mathrm{c}$ is the speed of light in vacuum [34].

Parallel to Heisenberg spatial uncertainty principle from Equation (12a),

$$
\Delta \lambda_{\beta} \Delta \overline{\mathrm{p}}_{\beta} \geq \hbar
$$

that limits the resolution of spatial measurements, Equation (12b) leads to temporal uncertainty principle [35]

$$
\Delta v_{\beta} \Delta \overline{\mathrm{p}}_{\beta} \geq \mathrm{k}
$$

that limits the resolution of time measurements. Implication of generalized uncertainty principle [87] to temporal uncertainty principle in Equation (13b) will be interesting. 
By Equation (12), photon internal or electromagnetic energy in Planck equilibrium radiation is expressed as

$$
\begin{aligned}
& \varepsilon_{\mathrm{rk}}=\mathrm{h} v_{\mathrm{rk}}=\mathrm{m}_{\mathrm{k}} \mathrm{v}_{\mathrm{rk}}^{2}=\mathrm{m}_{\mathrm{k}} \mathrm{c}^{2}=3 \mathrm{k} T \\
& \varepsilon_{\mathrm{rk}}=\mathrm{k} \lambda_{\mathrm{rk}}=\mathrm{m}_{\mathrm{k}} \mathrm{v}_{\mathrm{rk}}^{2}=\mathrm{m}_{\mathrm{k}} \mathrm{c}^{2}=3 \mathrm{k} T
\end{aligned}
$$

Hence, propagation speed of light is identified as root-mean-square speed of photons in Casimir [75] vacuum at EKD scale $\beta=\mathrm{k}$. This is in accordance with perceptions of Huygens [88] who believed that propagation of light waves in compressible ether is analogous to propagation of sound waves in compressible air. Because of the fundamental significance of this analogy to the gap between Planck equilibrium radiation theory and Boltzmann kinetic theory of ideal gas [36], the complete statement is quoted from Huygens book [88]

\begin{abstract}
"As regards the different modes in which I have said the movement of Sound and of Light are communicated, one may sufficiently comprehend how this occurs in the case of Sound if one considers that the air is of such nature that it can be compressed and reduced to a much smaller space than that which it ordinarily occupies.

Now in applying this kind of movement to that which produces Light there is nothing to hinder us from estimating the particles of the ether to be of a substance as nearly approaching to perfect hardness and pocessing a springiness as prompt as we choose. It is not necessary to examine here the causes of this hardness, or of that springiness, the consideration of which would lead us too far from our subject. I will say, however, in passing that we may conceive that the particles of the ether, notwithstanding their smallness, are in turn composed of the other parts and that their springiness consists in the very rapid movement of a subtle matter which penetrates them from every side and constrains their structure to assume such disposition as to give to this fluid matter the most overt and easy passage possible.This accords with the explanation which Mr. Des Cartes gives for the spring, though I do not, like him, suppose the pores to be in the form of round hollow canals. And it must not be thought that in this there is anything absurd or impossible, it being on the contrary quite credible that it is this infinite series of different sizes of corpuscles, having different degrees of velocity, of which Nature makes use to produce so many marvelous effects,"
\end{abstract}

It is most interesting that because of his deep appreciation of mathematics, Huygens kept an open mind concerning potential divisibility of atoms of ether.

At LKD (Figure 1) scale, Casimir [75] vacuum is considered to be a compressible tachyon fluid, Planck compressible ether [89] as discussed in [84]. Therefore, the most fundamental significance of Huygens [88] analogy between propagation of light and sound in the above quotation is the compressibility of physical space or Casimir [75] vacuum. Lorentz perceptions about the medium of space as Aristotle or Huygens ether [84] is described in the following quotation by Verhulst [90] from Lorentz 1915 lecture at the Royal Academy of Sciences in Amsterdam:

"Why can we not speak of the ether instead of vacuum? Space and time are not symmetric; a material point can at different times be at different spots, but not in different places at the same time"

Therefore, like sound waves, light waves have a longitudinal component or polarization associated with their gravitational mass through Higgs mechanism [35]. Space compressibility was shown [91] to account for causal nature of relativistic effects such as Lorentz-FitzGerald contraction when Michelson number $M i=\mathrm{v} / \mathrm{c}$ approaches unity thus leading to Poincaré-Lorentz dynamic theory of relativity as 
opposed to Einstein kinematic theory of relativity [91] in harmony with ideas of Darrigol [92] and Galison [93].

Although velocity of light is nearly constant, it is expected that it actually decreases extremely slowly, at cosmological time scale i.e., eons, with temperature of Casimir [75] vacuum due to expansion of the universe. Because of closure of the gap between radiation and gas theory [36], one could view Planck energy spectrum of equilibrium radiation as energy spectrum of Sackur "clusters" or Planck [94] "quantum sphere of action" as described by Darrigol [95-96] and in harmony with perceptions of de Broglie [36,97,98]

\section{"Existence of conglomerations of atoms of light whose movements are not independent but coherent"}

Hence, just like ideal gas, photon gas in Planck equilibrium radiation has granular structure and composed of ensembles of photon clusters with stationary energy, hence freuency (color), distribution.

The definition of Planck and Boltzmann universal constants in Equation (12) result in prediction of electromagnetic mass of photon, atomic-mass-unit $a m u$, Avogadro-Loschmidt number, and universal gas constant as [34]

$$
\begin{aligned}
& \mathrm{m}_{\mathrm{k}}=\left(\mathrm{hk} / \mathrm{c}^{3}\right)^{1 / 2} \approx 1.84278 \times 10^{-41} \mathrm{~kg} \\
& \text { amu }=\mathrm{m}_{\mathrm{k}} \mathrm{c}^{2}=(\mathrm{hkc})^{1 / 2} \approx 1.6563 \times 10^{-27} \mathrm{~kg} \\
& N^{\mathrm{o}}=1 /\left(\mathrm{m}_{\mathrm{k}} \mathrm{c}^{2}\right)=1 /(\mathrm{hkc})^{1 / 2} \approx 6.0376 \times 10^{26} \mathrm{kmol}^{-1} \\
& R^{\mathrm{o}}=N^{\mathrm{o}} \mathrm{k}=(\mathrm{k} / \mathrm{hc})^{1 / 2} \approx 8.338 \mathrm{~kJ} / \mathrm{kmol}-\mathrm{m}
\end{aligned}
$$

Because it is known that all baryonic matter is made of atoms, by Equation (17) all matter in the universe is composed of photon or electromagnetic mass in harmony with perceptions of Newton [99], Lorentz [100], and Poincaré [101-103].

By equation (15), Kelvin absolute temperature $T$ is identified as the most-probable or Wien wavelength $\lambda_{\mathrm{mp} \beta}=\lambda_{\mathrm{w} \beta}$ of particle thermal oscillations that is related to its de Broglie wavelength $\lambda_{\mathrm{r} \beta}=\lambda_{\mathrm{d} \beta}$ as $[33,34]$

$$
T_{\beta}=\left\langle\lambda_{\mathrm{mp} \beta}^{2}\right\rangle^{1 / 2}=\lambda_{\mathrm{w} \beta}=\lambda_{\mathrm{d} \beta} / 3
$$

Hence, zeroth law of thermodynamics of Fowler stating that equalities $T_{\mathrm{A}}=T_{\mathrm{B}}$ and $T_{\mathrm{A}}=T_{\mathrm{C}}$ require equality $T_{\mathrm{B}}=T_{\mathrm{C}}$, by Equation (20), gives $<\lambda_{\mathrm{wB}}^{2}>^{1 / 2}=<\lambda_{\mathrm{wC}}^{2}>^{1 / 2}$. Also, two kinds of "atomic" volume based on either Wien or de Borglie wavelengths [33]

$$
\hat{\mathrm{v}}_{\mathrm{w} \beta}=T_{\beta}^{3}=\lambda_{\mathrm{w} \beta}^{3} \quad, \quad \hat{\mathrm{v}}_{\mathrm{d} \beta}=\lambda_{\mathrm{d} \beta}^{3}
$$

lead to corresponding total volumes

$$
V_{\mathrm{w} \beta}=N_{\beta} \hat{\mathrm{v}}_{\mathrm{w} \beta} \quad, \quad V_{\mathrm{d} \beta}=N_{\beta} \hat{\mathrm{V}}_{\mathrm{d} \beta}
$$

where $V_{\mathrm{d}}=27 V_{\mathrm{w}}$. According to Equation (22), system volume increases either by increaseing temperature hence atomic volume in Equation (21) or by increasing the number of particles $N_{\beta}$. 
Increase of system volume with temerature in Equation (22) is consistent with observed operation of thermometers since most substances expand upon heating, exceept anomaly for water in the temperatures range $O<T<4{ }^{\circ} \mathrm{C}$. Also, since like photon gas, pressure and internal energy of ideal gas are related by $p_{\beta}=\tilde{\mathrm{u}}_{\beta} / 3$ [34], multiplication of Equation (22) with pressure results in ideal gas law

$$
p_{\beta} V_{\beta}=p_{\beta} N_{\beta} \hat{v}=\frac{\tilde{u}_{\beta} \hat{v}}{3} N_{\beta}=\frac{\hat{u}_{\beta}}{3} N_{\beta}=N_{\beta} \mathrm{k} T_{\beta}
$$

As described in [33, 34], because harmonic motion of oscillators are assumed to occur in two directions $\left(\mathrm{x}^{+}, \mathrm{x}^{-}\right)$, the classical and modified definitions of temperature and pressure are related by

$$
T^{\prime}=2 T \quad, \quad p^{\prime}=2 p
$$

The factor of 2 in Equation (24) leads to a modified expression for the speed of sound in standard atmosphere [57]

$$
\mathrm{c}_{\mathrm{s}}=\mathrm{v}_{\mathrm{rm}}=\sqrt{3 \mathrm{k} T / \mathrm{m}}=\sqrt{3 \mathrm{k} T^{\prime} /(2 \mathrm{~m})} \approx 343 \mathrm{~m} / \mathrm{s}
$$

in closer agreement with observed speed of sound than the classical Newton formula $c_{\mathrm{s}}^{\prime}=\sqrt{p^{\prime} / \rho}$. The result in Equation (25) is also in close agreement with Laplace's formula $c_{\mathrm{s}}^{\prime \prime}=\sqrt{\gamma \mathrm{k} T^{\prime}}$ with $\gamma=c_{p} / c_{v}$ denoting Poisson polytropic index. However, more recent investigation concerning physical foundation of Schrödinger equation [37] suggests that, as anticipated by Huygens [88] and described in the eloquent quotation above, analogous to the speed of light, the speed of sound is root-mean-square speed of molecules.

The results in Equation (15) regarding mass-energy equivalence and pioneering studies of Poincaré [101], de Pretto [104], Hasenöhrl [105], and Einstein [106] were discussed in an earlier study [34]. In the following we further examine the interesting but unfortunately little known study of Olinto de Pretto [104] who wrote:
"The formula $m v^{2}$ gives us $m v^{2} / 8338$ the living force and the formula gives us, expressed in calories, such energy.

Given that $m=1$ and $v$ equals three hundred thousand kilometers per second, that is 300 million meters, which would be the speed of light, also allowed for the ethere, everyone will see that you get an amount of calories represented by 10794 followed by 9 zeros and that is over ten million million."

The formula of de Pretto in the above quotation written in the present notation is

$$
\mathrm{mc}^{2} \mathrm{kcal}=\mathrm{mc}^{2} / 8338 \text { Joule }
$$

De Pretto number 8338 in Equation (26) was recently [107] identified as universal gas constant in Equation (19) expressed in MKS units

$$
R^{\mathrm{o}}=\mathrm{k} N^{\mathrm{o}}=8338 \text { Joule/kcal-m }
$$

Therefore, de Pretto Equation (26) shows that mechanical equivalent of heat should be numerically equal to $R^{\mathrm{o}}=8338$ Joule $/ \mathrm{kcal}-\mathrm{m}$. 
Accounting for the factor of 2 in definition of temperature $T_{\beta}^{\prime}=2 T_{\beta}$ from Equation (24), one finds the modified value of Joule-Mayer mechanical equivalent of heat

$$
J=2 J_{\mathrm{c}}=2 \times 4169=8338 \text { Joule } / \mathrm{kcal}
$$

where the value $J_{\mathrm{c}}=4.169 \approx 4.17 \mathrm{~kJ} / \mathrm{kcal}$ is the average of the two values $J_{\mathrm{c}}=(4.15,4.19)$ reported by Pauli [108]. Although $R^{\mathrm{o}}$ in Equation (27) and $J$ in Equation (28) are numerically equal, since their dimensions are different, this equality does not fully explain the nature of de Pretto Equation (26). This is because the same factor of 2 also occurs in the modified definition of pressure in Equation (24) making mechanical work on the left-hand-side of $-p V=-N k T$ to counterbalance the factor of 2 in temperature on its right-hand-side.

To reveal the true nature of mechanical equivalent of heat in de Pretto Equation (26), it is first noted that the universal gas constant may be expressed in terms of de Broglie wavelength from Equation (20) as

$$
R^{\mathrm{o}}=\mathrm{k} N^{\mathrm{o}}=\frac{\mathrm{k}}{\mathrm{m}_{\mathrm{k}} \mathrm{c}^{2}}=\frac{\mathrm{m}_{\mathrm{k}} \mathrm{v}_{\mathrm{rk}} \mathrm{c}}{\mathrm{m}_{\mathrm{k}} \mathrm{c}^{2}}=\frac{1}{\lambda_{\mathrm{rk}}}=\frac{1}{\lambda_{\mathrm{dk}}} \mathrm{m}^{-1}
$$

Next, for ideal gas and by Equation (20), equal amounts of energy are expressed in terms of atomic heat and work as [34]

$$
\begin{aligned}
& \hat{q}=Q / N=\hat{\mathrm{c}}_{\mathrm{v}} T=3 \mathrm{k} T=\hat{\mathrm{u}}=m_{\mathrm{k}} c^{2} \mathrm{kcal} \\
& \hat{w}=W / N=3 p \hat{\mathrm{v}}=3 \mathrm{k} T=3 \mathrm{k} \lambda_{\mathrm{w}}=\mathrm{k} \lambda_{\mathrm{d}}=m_{\mathrm{k}} c^{2} \text { Joule }
\end{aligned}
$$

Equations (29, 30a, 30b) give de Pretto Equation (26)

$$
\hat{q}=\mathrm{m}_{\mathrm{k}} c^{2} \mathrm{kcal}=\frac{\mathrm{k} \lambda_{\mathrm{d}}}{R^{\mathrm{o}}\left(\lambda_{\mathrm{d}}=1\right)}=\frac{\hat{w}}{J}=\frac{\mathrm{m}_{\mathrm{k}} c^{2}}{8338} \text { Joule }
$$

Thus, the definition of kcal as the unit of thermal energy is identified in

$$
J=R^{\mathrm{o}} \text { (Joule/kcal.m). }\left(\lambda_{\mathrm{d}}=1 \mathrm{~m}\right)=8338 \text { Joule/kcal }
$$

as energy per unit de Broglie temperature $T_{\mathrm{d}}=\lambda_{\mathrm{d}}=3 \lambda_{\mathrm{w}}=3 T=1 \mathrm{~m}$ corresponding to $p \hat{\mathrm{v}}=k T=\mathrm{k} / 3$, $\hat{\mathrm{u}}=3 \mathrm{k} T=\mathrm{k}$, and $\hat{\mathrm{h}}=4 \mathrm{k} T=4 \mathrm{k} / 3$. Multiplying Equation (31) by total number of particles $N$ gives the classical Joule-Mayer mechanical equivalent of heat

$$
W=J Q
$$

It is now clear that calculations based on the classical definition of temperature $T_{\beta}^{\prime}=2 T_{\beta}$ with the extra factor of 2 fortuitously compensated for the factor of $1 / 2$ in the classical Joule-Mayer mechanical equivalent of heat $J_{\mathrm{c}}=J / 2=4.169 \mathrm{~kJ} / \mathrm{kcal}$ thus resulting in agreement with empirical observations.

Since by Equation (20) temperature is identified as a metric of space, in a recent study [77] the concepts of internal spacetime versus external space and time were introduced. Assuming that a statistical field at scale $\beta$ is in thermodynamic equilibrium with the physical space at scale $(\beta-1)$ within which it resides, both fields will have a homogenous constant temperature $T_{\beta}=T_{\beta-1}$ defined as 
$\mathrm{m}_{\beta} \mathrm{u}_{\beta}^{2}=\mathrm{m}_{\beta-1} \mathrm{v}_{\mathrm{w} \beta-1}^{2}=\mathrm{k} T_{\beta-1}=\mathrm{k} \lambda_{\mathrm{w} \beta-1}$

Hence, by definition of most-probable or Wien speed $v_{w s}=\lambda_{w s} v_{w s}=\lambda_{w s} / \tau_{w s}$, one can associate constant internal measures of (extension, duration) [77]

$\begin{array}{ll}\lambda_{\mathrm{ws}} & \text { Internal measure of extension } \\ \tau_{\mathrm{ws}} & \text { Internal measure of duration }\end{array}$

with every "point" or "atom" of space at constant temperature $T_{\beta}=T_{\beta-1}$. Next, external space and time that are independent of each other are defined in terms of the internal spacetime as

$$
\left(\mathrm{x}_{\beta}, \mathrm{y}_{\beta}, \mathrm{z}_{\beta}\right)=\left(N_{\mathrm{x} \beta}, N_{\mathrm{y} \beta}, N_{\mathrm{z} \beta}\right) \lambda_{\mathrm{w} \beta-1} \quad, \quad t_{\beta}=N_{\mathrm{t} \beta} \tau_{\mathrm{w} \beta-1}
$$

with the four numbers $\left(N_{\mathrm{x} \beta}, N_{\mathrm{y} \beta}, N_{\mathrm{z} \beta}, N_{\mathrm{t} \beta}\right)$ being independent numbers. For example, at cosmic scale $\beta=g$, the internal (ruler, clock) of astrophysics $\beta=\mathrm{s}$ are employed to define external independent space and time coordinates by Equation (36) [77]. Therefore, internal spacetime in Equation (35) provides local structure of spacetime, whereas the external space and time in Equation (36) describe global dynamics of the system and are irreversible. According to Equations (35-36) both internal and external space and times are quantized. Irreversibility of space coordinates corresponds to noncommutative geometry [76] discussed in previous Section. The broad spectrum of physical time scales has been described in an excellent recent book [109]. The four dimensions $\left(\mathrm{x}_{\beta}, \mathrm{y}_{\beta}, \mathrm{z}_{\beta}, \mathrm{t}_{\beta}\right)$ with three real space and one imaginary time coordinates represent Poincaré [103] and Minkowski [110] fourdimensional spacetime manifold.

It was emphasized by Pauli [108] that time dimension does not occur in classical thermodynamics. However, according to Rovelli [111], thermodynamics plays a central role in the definition of physical time

"The very concept of time is meaningful only in the thermodynamic context"

The definition of internal spacetime in Equation (35) is based on thermodynamic equilibrium since the objective is to define what the variable called physical time represents as discussed by Rovelli [111]

"However, we are not concern here with versus of the time flow: we are concerned with definition of a variable that represents time"

Therefore, the external time quantitatively defined in Equation (36) is called Rovelli thermal time. It is ironic that the definition of physical time i.e. Rovelli thermal time must be based on systems that are at thermodynamic equilibrium hence stochastically stationary thus timeless. This is in harmony with the perceptions of both Aristotle [112] and St Augustine [113] who noted that the essence of time appears to be in what it is not. Of course, in all real processes dissipations lead to entropy generation resulting in change in temperature hence internal measures of spacetime in Equation (35). For example, in cosmology, the internal measure of spacetime change extremely slowly (eons) due to changes in Penzias-Wilson [114] cosmic background microwave radiation temperature of Casimir [75] vacuum $T_{\mathrm{CMB}} \approx 2.73 \mathrm{~m}$ caused by dissipations as well as expansion of the universe.

Since according to Equation (36), external (ruler, clock) $=\left(\mathrm{x}_{\beta}, \mathrm{t}_{\beta}\right)$ at scale $\beta$ are always defined in terms of internal spacetime $=\left(\lambda_{\mathrm{w} \beta-1}, \tau_{\mathrm{w} \beta-1}\right)$ at the next lower scale, the definition of (extension, duration) 
$=$ (space, time) can always be relegated to lower scales ad infinitum. This is because infinite divisibility of both extension and duration must follow the philosophy of Anaxagoras disccuessed by Bell [115]

"Neither is there a smallest part of what is small, but there is always a smaller, for it is impossible that what is should ever cease to be"

Hence, absolute zero and absolute infinite (extension, duration) are singularities and concieved as ideal Aristotle potential limits that strictly speaking are never actualized.

If one does not wish to allow for infinite divisibility of time, then following Leibniz [84] one must introduce the temporal monad just like the spatial monad to represent the absolute smallest "atom" of time or Chronon. The potentially infinite divisibility of time is related to the paradox of the present moment of time, instanton [116] or "now" that separates two non-existent worlds, a past already lived and a future yet to come. Poincaré gives an eloquent description of the mystery of life through "now" at the very end of his wonderful book [117]

"And yet--strange contradictions for those who believe in time--geologic history shows us that life is only a short episode between two eternities of death, and that, even in this episode, conscious thought has lasted and will last only a moment. Thought is only a gleam in the midst of a long night. But it is this gleam which is everything."

It is interesting that an instant of time in cosmology was estimated [77] as 100,000 years, namely the time it takes to cross the Milky Way travelling at the speed of light.

Because of change of units $\mathrm{m} / \mathrm{cm}=100$ required by Wien displacement law $\lambda_{\mathrm{w}} T=0.29 \mathrm{~cm} . \mathrm{K}=0.0029 \mathrm{~m}^{2}$, the classical formula for temperature conversion $T[\mathrm{~K}]={ }^{\circ} \mathrm{C}+273.1$ is modiefied as

$$
T(\mathrm{~m})={ }^{\circ} \mathrm{C}(\mathrm{m})+2.731(\mathrm{~m})
$$

It is important to note that the constant $2.731 \mathrm{~m}$ in Equation (37) is close to Penzias-Wilson [114] cosmic microwave background radiation temperature $T_{\mathrm{CMB}} \approx 2.73 \mathrm{~m}$.

It is also interesting to examine implication of change of dimension of temperature to the physical meaning of Planck [118] temperature

$$
T_{\mathrm{p}}=\left(\mathrm{hc}^{5} / \mathrm{Gk}^{2}\right)^{1 / 2} \approx 3.55 \times 10^{32} \mathrm{~m}
$$

as cosmic temperature at the initial explosion of Lemaitre [119] "primordial atom" of our universe. Because by Equation (20) absolute temerature is the most-probable or Wien wavelength of thermal oscillations $T_{\beta}=\lambda_{\mathrm{w} \beta}$, Planck temperature in Equation (38) is puzzling since it is much larger than the size of our universe $L_{\mathrm{g}} \approx 10^{26} \mathrm{~m}$ reported in the literature. Based on known approximate length scales of our uinverse, and the scale facor of $10^{17}$ in Figure 3, one may consider the (atom, element, system) $=$ $\left(\ell_{\beta}, \lambda_{\beta}, L_{\beta}\right)$ lengths,

EGD $\quad\left(\ell_{\mathrm{g}}, \lambda_{\mathrm{g}}, \mathrm{L}_{\mathrm{g}}\right)=\left(10^{18}, 10^{26}, 10^{35}\right) \mathrm{m}$

$\operatorname{EUD} \quad\left(\ell_{\mathrm{u}}, \lambda_{\mathrm{u}}, \mathrm{L}_{\mathrm{u}}\right)=\left(10^{26}, 10^{35}, 10^{43}\right) \mathrm{m}$

repectively corresponding to cosmology or equilibrium galactic-dynamics EGD at scale $\beta=\mathrm{g}$ (Figure 1) and the next major statistical field called equilibrium-universal-dynamics EUD $\beta=\mathrm{u}$ ( not shown in Figure 1), in harmony with many-uinverse concept of Everett [120]. Hence, what is believed to be 
the present size of our universe appears to correspond to the visible horizon associated with the mostprobable galactic-cluster in a much larger ultra- cosmic field required in order to accommodate photons with mean-free-paths as long as $T_{\mathrm{g}}=T_{\mathrm{s}}=\lambda_{\text {wg }}$ in Equation (38). Clearly, this result enhances the probability of existence of multiverse in harmony with the hierarchy shown in Figure 1, the perceptions of Everett [120], as well as inflationary models of cosmology [121-124].

\section{Helmholtz Decomposition of Energy and the First Law of Thermodynamics}

The empirical foundation of principle of conservation of energy or the first law of thermodynamics was based on experimental studies by Mayer and Joule focused on mechanical equivalent of heat and performance of heat engines compared to ideal Carnot cycle. Therefore, classical form of the first law of thermodynamics $[108,125]$

$$
d E=\delta Q-\delta W
$$

expresses the change of system energy as a result of heat added to the system and work done by the system

\section{Total energy inside system $=$ Heat input - Work output}

However, it was emphasized by Warner [126] that the form in Equation (40) is only applicable to reversible processes and some authors have instead used an alternative formulation

$$
d E=\delta Q+\delta W^{\prime}
$$

where $\delta W^{\prime}$ is the work done on the system by external forces that are not derivable from a potential, and $d E$ and $\delta Q$ retain the same meaning as in Equation (40).

On the other hand, theoretical foundation of the first law of thermodynamics was first introduced by Helmholtz [127] and Clausius [128, 129] through application of virial theorem to weakly interacting particles such as ideal gas. Helmholtz in his pioneering 1847 paper concerning conservation of energy argued that the quantity of heat or thermal energy of the system may be decomposed into two parts respectively associated with the kinetic energy or vis viva of particles and potential energy due to the stress or pressure field caused by their mutual interactions [127]

"That which has been heretofore named the quantity of heat, according to this, be the expression, first, of the quantity of vis viva of the caloric motion, and secondly, of the quantity of those tensions between the atoms, which, by changing the arrangement of the latter, such a motion can develop. The first portion would correspond to that which has been heretofore called free heat, the second with that which has been named latent heat".

Following Helmholtz [127], the first law of thermodynamics is expressed as [33,34],

$$
\text { System total thermal energy }=\text { Free heat }+ \text { Laten heat }
$$

When free heat and latent heat are respectively identified as internal energy $U$ and potential energy $p V$, such that total thermal energy of the system becomes enthalpy $H=Q$, and work is identified as Helmholtz free energy $W=F=U-T S=-p V$, by Equation (42), the modified form of the first law of thermodynamics becomes $[33,34]$

$$
d Q=d H=d(T S)=d U-d F=d U-d W=d U+d(p V)
$$

with reversible heat and reversible work defined as 


\section{$W=F=-p V \quad$ Reversible work}

In Equation (45), the choice of negative sign of reversible work or Helmholtz free energy $W=F=U-T S=-p V$ ensures that work done on the system by compression thus reducing its volume $V_{2}\left\langle V_{1}\right.$ will be positive $W=-p\left(V_{2}-V_{1}\right)>0$ as required. The first law of thermodynamics in Equations (43-45) that is based on perceptions of Helmholtz [127] requires new scientific paradigms involving a modified mechanical theory of heat as well as a new definition of potential energy that are described in the following.

As discussed in $[33,34]$, according to Equations $(1,3)$, particle translational velocity is the sum of the mean or "cluster" velocity and its peculiar velocity

$$
\mathbf{u}_{\mathrm{mj}}=\mathbf{v}_{\mathrm{mj}}+\mathbf{V}_{\mathrm{mj}}^{\prime}
$$

The inclusion of peculiar velocity is of central importance since it defines what is called potential energy of particle. For example, in cosmology, peculiar velocity of a galaxy is the difference between its velocity and the velocity of the cluster of galaxies to which it belongs. In ideal gas at thermodynamic equilibrium, all three velocities in Equation (46) will be random such that Equation (46) after being squared, averaged, and multiplied by particle mass leads to kinetic and potential energies [34]

$$
\begin{aligned}
& \hat{u}_{\mathrm{t}}=\frac{1}{2} \mathrm{~m}_{\mathrm{v}_{\mathrm{wmx}+}^{2}}+\frac{1}{2} \mathrm{~m} \overline{\mathrm{v}_{\mathrm{wmx}-}^{2}}=\frac{1}{2} \overline{\mathrm{mu}_{\mathrm{cx}+}^{2}}+\frac{1}{2} \mathrm{mu}_{\mathrm{cx}-}^{2}=\mathrm{mv}_{\mathrm{wmx}+}^{2} \\
& \hat{\varepsilon}_{\mathrm{p}}=\frac{1}{2} m \overline{\mathrm{V}_{w m x+}^{\prime 2}}+\frac{1}{2} m \overline{\mathrm{V}_{w m x-}^{\prime 2}}=m \overline{\mathrm{V}_{w m x+}^{\prime 2}}=\frac{1}{3} m \overline{\mathrm{V}_{w m+}^{\prime 2}}=p \hat{\mathrm{v}}
\end{aligned}
$$

with pressure defined as $p=\mathrm{nm}_{\mathrm{V}_{\mathrm{wm}+}^{\prime 2}} / 3$. Therefore, the total energy due to harmonic translational motion of particle called atomic translational emthalpy becomes

$$
\hat{h}_{t}=\hat{u}_{t}+\hat{\varepsilon}_{\mathrm{p}}=\hat{u}_{t}+p \hat{\mathrm{v}}=2 \mathrm{k} T
$$

As discussed in [33, 34], the conventional practice of attributing identical translational kinetic energy $\left(\hat{u}_{\mathrm{tx}}, \hat{u}_{\mathrm{ty}}, \hat{u}_{\mathrm{tz}}\right)$ to all three coordinate directions $(\mathrm{x}, \mathrm{y}, \mathrm{z})$ is not appropriate because a particle cannot simultaneously move along three independent coordinate directions. In addition, as was emphasized by Clausius [128], rotational and vibrational energy of particles cannot be properly neglected

"In liquids, therefore, an oscillatory, a rotatory, and a translator motion of the molecules take place, but in such a manner that these molecules are not themselves separated from each other, but even in the absence of external forces, remain within a certain volume"

Following Clausius $[128,129]$, harmonic translational kinetic energy in $\left(\mathrm{y}^{+}, \mathrm{y}^{-}\right)$and $\left(\mathrm{z}^{+}, \mathrm{z}^{-}\right)$directions are instead attributed to kinetic energies due to particles harmonic rotational $\left(\theta^{+}, \theta^{-}\right)$and vibrational $\left(\mathrm{r}^{+}, \mathrm{r}^{-}\right)$motions in two coordinate directions [34]

$$
\hat{u}_{\mathrm{r}}=\varepsilon_{\mathrm{r}}=\frac{1}{2} I \overline{\omega_{\mathrm{m} \theta+}^{2}}+\frac{1}{2} I \overline{\omega_{\mathrm{m} \theta-}^{2}}=I \overline{\omega_{\mathrm{m} \theta+}^{2}}
$$


$\hat{u}_{\mathrm{v}}=\varepsilon_{\mathrm{v}}=\frac{1}{2} \chi \overline{\mathrm{r}_{\mathrm{m}+}^{2}}+\frac{1}{2} \chi \overline{\mathrm{r}_{\mathrm{m}-}^{2}}=\chi \overline{\mathrm{r}_{\mathrm{m}+}^{2}}$

with $(I, \chi)$ respectively denoting particle moment of inertia and spring constant.

Next, total atomic internal energy is defined as the sum of translational, rotational, and vibrational atomic internal energies from Equations (47), (50), and (51) as

$$
\hat{u}=\hat{u}_{t}+\hat{u}_{r}+\hat{u}_{v}=m \overline{v_{x+}^{2}}+I \overline{\omega_{x+}^{2}}+\chi r_{x+}^{2}
$$

Since Boltzmann principle of equipartition of energy requires all four degrees of freedom to have the same energy

$$
\hat{u}_{t}=\hat{u}_{r}=\hat{u}_{v}=p \hat{\mathrm{v}}=\mathrm{k} T
$$

atomic enthaply becomes

$$
\hat{h}=\hat{u}+p \hat{\mathrm{v}}=4 \mathrm{k} T
$$

Multiplication of Equation (54) with number of atoms $N$ results in what Sommerfeld [125] called total heat or enthalpy

$$
H=U+p V
$$

As emphasized by Pauli [108],

"In thermodynamics no considerations concerning the nature of heat are made. This problem is first dealt with in the kinetic theory of gases."

The fact that a satisfactory mechanical theory of heat in thermodynamics was not developed since the pioneering work of Helmholtz [127] could be in part due to the neglect of peculiar velocity of particles in Equation (46). Since peculiar velocity represents an independent translational degree of freedom, associated with particle potential energy in Equation (48) hence Poincaré stress [35, 37], without its inclusion in the kinetic theory of gases a satisfactory mechanical theory of heat cannot be realized. Equations (53)-(55) constitute a "derivation" of the first law of thermodynamics and the state function $H$, named enthalpy by Onnes and called "heat function" or "total heat" by Sommerfeld [125], can be identified as the mechanical theory of heat in thermodynamics that was called for in the above quotation from Pauli [108].

The first law of thermodynamics in Equation (43) can be directly derived from classical results [125] by addition of Gibbs equation

$$
T d S=d U+p d V-\sum_{\mathrm{i}} \mu_{\mathrm{i}} d N_{\mathrm{i}}
$$

and Gibbs-Duhem equation

$$
S d T=V d p-\sum_{\mathrm{i}} N_{\mathrm{i}} d \mu_{\mathrm{i}}
$$

hence

$$
d(S T)=d U+d(p V)-\sum_{\mathrm{i}} d\left(N_{\mathrm{i}} g_{\mathrm{i}}\right)
$$


In non-reactive systems $d N_{\mathrm{i}}=0$, Equation (57) reduces to Equation (43) since Gibbs chemical potential $\mu_{\mathrm{i}}=g_{\mathrm{i}}$ of ideal gas is zero $[33,34]$

$$
\mu_{\mathrm{i}}=g_{\mathrm{i}}=\left(\partial \mathrm{U} / \partial N_{\mathrm{i}}\right)_{\mathrm{S}, \mathrm{N}, \mathrm{j} \neq \mathrm{i}}=0
$$

Modified form of the first law of thermodynamics in Equation (43) is to be compared with the classical form [108, 125]

$$
d U=\delta Q-\delta W
$$

with heat $\delta Q$ and work $\delta W$ that are path-dependent hence not state functions. Since both reversible heat and work in Equations, (44)-(45) are state functions, the paradox of exact cancellation of pathdependence of heat and work postulated in classical thermodynamics $[108,125]$

$$
\int(\delta Q-\delta W)=0
$$

in order to obtain an exact differential $d U$ in Equation (59) is resolved.

To reveal the origin of path-dependence one notes that both reversible heat $Q(T, S)=T S$ and reversible work $W(p, V)=-p V$ depend on two independent variables hence the total differentials

$$
d Q=T d S+S d T \quad, \quad d W=-p d V-V d p
$$

Because reversible heat and work transfer are achieved respectively under isothermal $S d T=0$ and isobaric $V d p=0$ conditions, the last terms in both parts of Equation (61) become identically zero. If, however, such reversibility conditions are not satisfied but the last terms in Equation (61) are nevertheless neglected, then differentials $\delta Q_{\text {irr }}$ and $\delta W_{\text {irr }}$ will no longer be exact and become pathdependent.

For an ideal gas, enthalpy $H=4 N \mathrm{k} T=S T$, internal energy $U=3 N \mathrm{k} T=(3 / 4) T S$, and work or potential energy $W=-P V=-N \mathrm{k} T=-T S / 4$ are all state functions involving Boltzmann constant $\mathrm{k}$ that always occurs with absolute temperature due to the definition of atomic energy $\mathrm{k} T_{\beta}=\hat{\varepsilon}_{\beta}$. However, the universal constant $\mathrm{k}$, first calculated by Planck and named Boltzmann constant, also occurs in Boltzmann formula for entropy $S=\mathrm{k} \ln \mathrm{W}$. Therefore, to follow the notation of classical thermodynamics, in the definitions of $(H, U, W)$ above, one groups $\mathrm{k}$ with $4 N$ in the definition of entropy $S=4 \mathrm{kN}[33,34]$ rather than with $T$ in the definition of "atomic" energy $\mathrm{k} T=\hat{\varepsilon}$.

On a related subject matter concerning exact differentials, because of the modified definition of velocity potential $\mathbf{v}=-\nabla \Phi[35,37]$, the complex velocity potential becomes

$$
\mathrm{Z}(\mathrm{z})=\tilde{\Psi}(\mathrm{x}, \mathrm{y})+\mathrm{i} \Phi(\mathrm{x}, \mathrm{y})
$$

and velocity potential $\Phi$ rather than stream function $\tilde{\Psi}$ becomes the imaginary part of Z(z) such that with complex variable $\mathrm{Z}=x+$ iy , Cauchy-Riemann conditions for analyticity of $\mathrm{Z}(\mathrm{z})$ become

$$
\Phi_{\mathrm{x}}=-\tilde{\Psi}_{\mathrm{y}} \quad, \quad \Phi_{\mathrm{y}}=\tilde{\Psi}_{\mathrm{x}}
$$

This modification is important because according to invaraint Schrödinger equation [37], quantum mechanics wave function $\Psi$ is related to the velocity potential of particle peculier motion $\Phi^{\prime}$. Thus, in harmony with perceptions of de Broglie [3], the complex wave function $\Psi$ guides the motion of particle that is a singularity, i.e., wave-packet at lower scale $\beta-1$, on this wave [37]. Since $\Phi^{\prime}, \tilde{\Psi}$, and Z satisfy Cauchy-Riemann conditions in Equation (63), they are all harmonic functions that satisfy 
Laplace equation and hence wave equations. Stationary waves of $T, \rho$, and $p$ lead to waves of energy $(H, U, W)$ associated with stationary states corresponding to solutions of time-independent Schrödinger equation as described in [37].

Since according to Gibbs's phase rule, given any state function $H, U, \ldots$ the state of a pure substance is defined by the remaing two thermdynamic variables, one introduces the complex variable $\mathrm{z}=T+\mathrm{i} S$ with the imaginary axis corresponding to entropy. Because of virtual nature of HeisenberyKramers [51] oscillators, associating imaginary numbers with $S$ rather than $T$ is reasonable. Thus, with vertical axis corresponding to complex part of $\mathrm{z}=T+\mathrm{i} S$, Carnot cycle is shown as $(S-T)$ diagram in Figure 6.

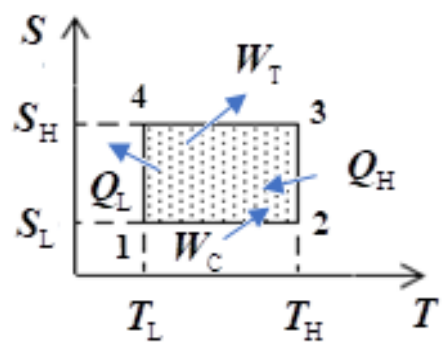

Figure 6. Carnot cycle.

For an ideal gas, the total system thermal energy $H=S T=4 \mathrm{kNT}$ can change by either changing the number of oscillators $S=4 \mathrm{kN}$, or by changing the energy per oscillator $\hat{\varepsilon}=\mathrm{k} T$ hence $T$, or both. In Figure 6, heating $2 \rightarrow 3$ and cooling $4 \rightarrow 1$ processes in boiler and condenser occur isothermally through increase and decrease of the number of oscillators $\Delta Q=(4 \mathrm{k} T) \Delta N$ such as occur by evaporation and condensation during change of phase. On the other hand, work transfer in turbine $3 \rightarrow 4$ and compressor $1 \rightarrow 2$ occur at constant entropy (isentropic) by changing temperature $\Delta W=-\mathrm{k} N \Delta T$ due to cooling and heating of the working fluid by expansion and compression. It is noted that isentropic change of volume $V=N \hat{\mathrm{v}}$ during expansion $3 \rightarrow 4$ or compression $1 \rightarrow 2$ occur through changes of temperature hence atomic volume $\hat{\mathrm{v}}=T^{3}=\lambda_{\mathrm{w}}^{3}$ [33] rather than $S$ hence number of virtual oscillators $N$.

Closure of the gap between Planck theory of equilibrium photon gas and Boltzmann kinetic theory of ideal gas [36], requires the total energy of a system composed of either gas be the enthalpy $H$ rather than the internal energy $U$ as reported in some literature. To show this, one notes that for equilibrium radiation, the number of photons in volume $V$ of Casimir [75] vacuum is given as [36]

$$
N=\frac{8 \pi^{5} V}{45}\left(\frac{\mathrm{k} T}{\mathrm{hc}}\right)^{3}
$$

Integration of Planck energy distribution in Equation (6) for equilibrium photon gas over the total frequency range $v=(0 \rightarrow \infty)$ gives

$$
<E>=T^{\prime} S=\frac{8 \pi^{5} V}{90} \frac{\mathrm{k}^{4} T^{\prime 4}}{\mathrm{~h}^{3} \mathrm{c}^{3}}
$$

Substitutions for $T^{\prime}=2 T$ and $N$ from Equations (24) and (64) in Equation (65) give

$$
H=T S=\frac{4}{3}\left\{3\left[\frac{8 \pi^{5} V}{45}\left(\frac{\mathrm{k} T}{\mathrm{hc}}\right)^{3}\right] \mathrm{k} T\right\}=\frac{4}{3}(3 N \mathrm{k} T)=\frac{4}{3} U=4 N \mathrm{k} T
$$


The result in Equation (66) is in agreement with the relation between total energy and electromagnetic energy of photon predicted by Hasenöhrl $[105,130]$

$$
H_{\beta}=\mathrm{M}_{\mathrm{t}} \mathrm{c}^{2}=\frac{4}{3} \mathrm{M}_{\mathrm{EM}} \mathrm{c}^{2}
$$

and relates photon total mass and electro-magnetic mass [37] as

$$
M_{\mathrm{t}}=\frac{4}{3} \mathrm{M}_{\mathrm{EM}}
$$

Since the prediction of Hasenöhrl [105,130] in Equation (67) is for equilibrium radiation in enclosures, photons will be under stationary-state and must oscillate at the most probable or Wien speed

$$
\mathrm{v}^{2}=\mathrm{v}_{\mathrm{kw}}^{2}=\mathrm{v}_{\mathrm{kw}+}^{2}+\mathrm{v}_{\mathrm{kw}-}^{2}=2 \mathrm{v}_{\mathrm{kw}+}^{2}
$$

Substituting for $\mathrm{v}^{2}$ from Equation (69) in Lorentz [100] relativistic mass formula

$$
\mathrm{m}_{\mathrm{r}}=\mathrm{m}_{\mathrm{o}} / \sqrt{1-\mathrm{v}^{2} / \mathrm{c}^{2}}
$$

results in

$$
\mathrm{m}_{\mathrm{rk}}=\mathrm{m}_{\mathrm{ok}}\left(1+\mathrm{v}_{\mathrm{kw}}^{2} / 2 \mathrm{c}^{2}\right)=\left(1+\mathrm{v}_{\mathrm{kw}+}^{2} / \mathrm{c}^{2}\right)=\mathrm{m}_{\mathrm{ok}}(1+1 / 3)=(4 / 3) \mathrm{m}_{\mathrm{k}}
$$

in accordance with Equations $(67,68)$.

The above results resolve the classical problem of total radiant energy under Planck distribution function being internal energy $\langle E\rangle=U$ instead of the enthalpy $\langle E\rangle=H$. Interestingly, the correct relationship between enthalpy $H=T S$ and internal energy $U$ in Equation (67) also appear in the books by both Planck [118] as well as Sommerfeld [125]. However, classical expressions for the number of photons in vacuum instead of the result in Equation (64) do not lead to the correct total energy $H$ under Planck distribution curve given in Equation (66).

The result in Equation (71) is of fundamental significance because exactly $3 / 4$ and $1 / 4$ of the total thermal energy under Planck [94] distribution curve fall on $v>v_{w}$ and $v<v_{w}$ sides of Wien frequency $v_{w}[77]$ as shown in Figure 7.

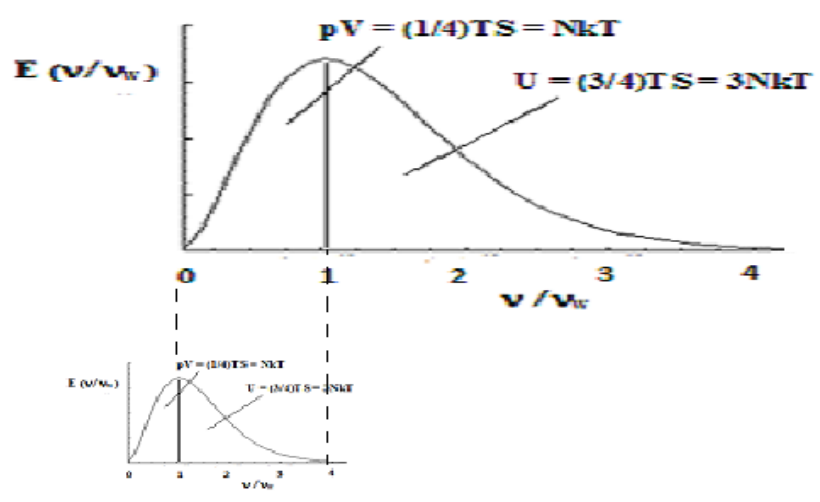

Figure 7. Re-normalized Planck energy distribution as a function of $\mathrm{V} / \mathrm{v}_{w}$ [77]

For an ideal gas the potential and internal energy are related by [33, 34]

$$
p_{\beta} V_{\beta}=\frac{U_{\beta}}{3}=\frac{3 N \mathrm{k} T_{\beta}}{3}=N \mathrm{k} T_{\beta}
$$


From Equation (55), enthalpy and internal energy are related by

$$
H_{\beta}=U_{\beta}+\frac{U_{\beta}}{3}=U_{\beta}+\frac{H_{\beta}}{4}=U_{\beta}+\frac{1}{4}\left(U_{\beta}+\frac{U_{\beta}}{3}\right)=\ldots=U_{\beta}\left(1+\frac{1}{4}+\frac{1}{4^{2}}+\frac{1}{4^{3}}+\ldots\right)=\frac{4}{3} U_{\beta}
$$

Since according to Figure 7, potential energy of scale $\beta$ is identified as the total thermal energy or enthalpy of the lower scale $\beta-1$ [33]

$$
D M_{\beta}=p_{\beta} V_{\beta}=\frac{H_{\beta}}{4}=H_{\beta-1}
$$

total system energy in Equation (73) may be expressed as

$$
H_{\beta}=U_{\beta}+\frac{H_{\beta}}{4}=U_{\beta}+H_{\beta-1}=U_{\beta}+U_{\beta-1}+\frac{H_{\beta-1}}{4}=\ldots=\sum_{\mathrm{n}=0} U_{\beta-\mathrm{n}}
$$

Applying Helmholtz decomposition at cosmic scale $\beta=\mathrm{g}$, of the total thermal energy $H$ of the universe, $3 / 4$ is free heat or dark-energy and is associated with electromagnetic mass, and $1 / 4$ is laten heat or dark-matter and is associated with gravitational mass [33, 131-135]. The $3 / 4$ and $1 / 4$ division of energy in Equations (67) and (74) is also in accordance with recent observations [136-139] and general theory of relativity [140-141] as described by Pauli [141]

"The energy of a spatially finite universe is three-quarters electromagnetic and one-
quarter gravitational in origin"

Scale invariance of the model suggests that dark matter in Equation (74) may be expressed as [33]

$$
D M_{\beta+1}=H_{\beta}=U_{\beta}+p_{\beta} V_{\beta}=D E_{\beta}+D M_{\beta}=D E_{\beta}+D E_{\beta-1}+D M_{\beta-2}=\ldots=\sum_{\beta}^{\beta-\infty} D E_{\beta}
$$

By Equations $(18,74,76)$, all baryonic matter in the universe is made of dark energy or electromagnetic mass as anticipated by Newton [99], Lorentz [100] and Poincaré [101-103].

\section{Clausius, Boltzmann, and the Second Law of Thermodynamics}

In classical thermodynamics, definition of entropy introduced by Clausius is based on reversible heat as

$$
d S=\frac{d Q_{\mathrm{rev}}}{T}
$$

In view of Equation (43) and reversible heat in Equation (44), entropy of ideal gas is directly related to enthalpy by

$$
S=\frac{Q_{\text {rev }}}{T}=\frac{H}{T}
$$

Hence, entropy of an ideal gas by Equations (77) and (78) becomes [33, 34]

$$
S=4 \mathrm{k} N
$$

The direct connections of entropy $S$ and total heat or enthalpy $H$ hence the number of HeisenbergKramers [51] virtual oscillators reveal its objective rather than subjective nature as discussed in [142] in connection to Shannon information theory.

For isolated systems, constant total system energy gives

$$
d Q=d H=d(T S)=0
$$


leading directly to the second law of thermodynamics

$T d S=-S d T \geq 0$

Since by Clausius statement of second law, heat must always spontaneously flow from high to low temperature $T_{\mathrm{H}} \rightarrow T_{\mathrm{L}}$, one must have $d T \leq 0$ leading to Equation (81). Therefore, in isolated systems ordered (correlated) motions transform, hence the name entropy chosen by Clausius [129], into disordered (un-correlated or random) motions leading to increase of system entropy $d S=4 \mathrm{k} d N \geq 0$ by Equation (81). As emphasized by Boltzmann [143], the reversal of the process hence de-randomization of microscopic thermal motions back into macroscopic ordered motions will be extremely unlikely bordering impossibility. It is well known that Eddington arrow of time arises in all real hence noneequilibrium processes and is directly connected to irreversibility associated with increase of entropy by dissipative processes $[35,36,77,144]$. The Zemerlo-Boltzmann debates concerning reversibility and Poincaré recurrence theorem were discussed in [36].

The statistical nature of entropy is based on the fundamental question asked by both Boltzmann and Planck namely: given the total energy $H$ and the total number of particles or oscillators $N$, what is the distribution of numbers $N_{\mathrm{j}}$ of indistinguishable particles placed in $g_{\mathrm{j}}$ distinguishable boxes or "clusters" in order to achieve equilibrium speed (Maxwell-Boltzmann) and energy (Planck) distributions. Of course, the particles are under constant motion by transition between clusters that are stochastically stationary by the principle of detailed balance. Clearly, this question directly relates to the problems of kinetic theory of ideal gas of Boltzmann [143] and theory of equilibrium radiation of photon gas of Planck [94]. Boltzmann anticipated quantum mechanics by about three decades as evidenced by the following quotation taken from his pioneering and often neglected 1872 paper [145],

"We wish to replace the continuous variable $x$ by a series of discrete values $\varepsilon, 2 \varepsilon, 3 \varepsilon, \ldots p \varepsilon$. Hence, we must assume that our molecules are not able to take up a continuous series of kinetic energy values, but rather only values that are multiples of a certain quantity $\varepsilon$. Otherwise, we shall treat exactly the same problem as before. We have many gas molecules in a space $R$. They are able to have only the following kinetic energies:

$$
\varepsilon_{\mathrm{j}}, 2 \varepsilon_{\mathrm{j}}, 3 \varepsilon_{\mathrm{j}}, 4 \varepsilon_{\mathrm{j}}, \ldots . \mathrm{p} \varepsilon_{\mathrm{j}}
$$

No molecule may have an intermediate or greater energy. When two molecules collide, they can change their kinetic energies in many different ways. However, after the collision the kinetic energy of each molecule must always be a multiple of E. I certainly do not need to remark that for the moment we are not concerned with a real physical problem. It would be difficult to imagine an apparatus that could regulate the collisions of two bodies in such a way that their kinetic energies after a collision are always multiples of $\varepsilon$. That is not a question here."

Both Boltzmann [143, 145] and Planck [94] applied combinatorics to arrive at the number of what Boltzmann called "complexions" for distributing $N \mathrm{j}$ indistinguishable particles among $g_{\mathrm{j}}$ distinguishable boxes or cells

$$
\mathrm{W}_{\mathrm{j}}=\frac{\left(N_{\mathrm{j}}+g_{\mathrm{j}}-1\right) !}{N_{\mathrm{j}} !\left(g_{\mathrm{j}}-1\right) !} \quad, \quad \mathrm{W}=\prod_{\mathrm{j}} \mathrm{W}_{\mathrm{j}}
$$

When number of boxes or cells $g_{\mathrm{j}}$ is viewed as number of particle "clusters", because the smallest cluster can contain only a single particle, the number of available clusters or quantum states will be $\left(g_{\mathrm{j}}-1\right)$. As a result, the inverse of Planck- Boltzmann Equation (82) is exactly the probability of distributing $N_{\mathrm{j}}$ indistinguishable oscillators amongst $\left(g_{\mathrm{j}}-1\right)$ distinguishable available clusters [33, 34], 
$\mathrm{p}_{\mathrm{j}}=\frac{N_{\mathrm{j}} !\left(g_{\mathrm{j}}-1\right) !}{\left(N_{\mathrm{j}}+g_{\mathrm{j}}-1\right) !}=\frac{1}{\mathrm{~W}_{\mathrm{j}}} \quad, \quad \mathrm{p}=\prod_{\mathrm{j}} \mathrm{p}_{\mathrm{j}}=\frac{1}{\mathrm{~W}}$

In the realistic limit $g_{\mathrm{j}}>>N_{\mathrm{j}}>>1$ all three statistical fields namely Boltzmann, Fermi-Dirac, and Bose-Einstein statisitcs reduce to what is called corrected Boltzmann statistics $[55,56]$

$$
\mathrm{W}_{\mathrm{j}}=\frac{g_{\mathrm{j}}^{N_{\mathrm{j}}}}{N_{\mathrm{j}} !}
$$

Substituting for $g_{\mathrm{j}}$ in terms of the volume $V_{\mathrm{j}}$ of cluster $\mathrm{j}$ and its atomic volume $\hat{\mathrm{v}}_{\mathrm{j}}=T^{3}=\lambda_{\text {wj }}^{3}$ the number of complexions in Equation (84) simplifies to

$$
\mathrm{W}_{1 \mathrm{j}}=\frac{\left(V_{\mathrm{j}} / \lambda_{\mathrm{wj}}^{3}\right)^{N_{\mathrm{j}}}}{N_{\mathrm{j}} !}=\frac{N_{\mathrm{j}}^{N_{\mathrm{j}}}}{N_{\mathrm{j}} !} \approx \mathrm{e}^{N_{\mathrm{j}}} \quad, \quad \mathrm{W}_{1}=\prod_{\mathrm{j}} \mathrm{W}_{1 \mathrm{j}} \approx \mathrm{e}^{N}
$$

that is in accordance with partition function $\mathrm{Z}$ of an ideal gas given by Kardar [56]. Next, one accounts for four degrees of freedom associated with translational, rotational, vibrational, and potential energy of each oscillator and by (85) and Boltzmann equipartition principle the total numbers of complexions become $[33,34]$

$$
\mathrm{W}_{\mathrm{j}}=\mathrm{W}_{4 \mathrm{j}}=\mathrm{W}_{\mathrm{tj}} \mathrm{W}_{\mathrm{rj}} \mathrm{W}_{\mathrm{vj}} \mathrm{W}_{\mathrm{pj}}=\mathrm{e}^{4 N_{\mathrm{j}}} \quad, \quad \mathrm{W}=\prod_{\mathrm{j}} \mathrm{W}_{4 \mathrm{j}} \approx \mathrm{e}^{4 N}
$$

In view of Equation (83) Boltzmann [143, 145] formula for entropy expressed in terms of probabilities is [33],

$$
S_{\mathrm{j}}=-\mathrm{k} \ln \mathrm{p}_{\mathrm{j}} \quad, \quad S=\sum_{\mathrm{j}} S_{\mathrm{j}}=-\mathrm{k} \ln \mathrm{p}
$$

Following Boltzmann [143, 145], one introduces "atomic" probability as

$$
\hat{\mathrm{p}}=\mathrm{e}^{-\hat{h} / \mathrm{k} T}
$$

By definition of atomic enthalpy in Equation (54) and Equations (86)-(88), entropy of "atom", cluster$\mathrm{j}$, and system become $[33,34]$

$$
\begin{array}{ll}
\hat{s}=-\mathrm{k} \ln \hat{\mathrm{p}}=4 \mathrm{k} \quad, \quad \hat{\mathrm{p}}=\mathrm{e}^{-\hat{h} / \mathrm{k} T}=\mathrm{e}^{-4} \\
S_{\mathrm{j}}=\sum_{\mathrm{j}} \hat{s}=-\mathrm{k} \ln \mathrm{p}_{\mathrm{j}}=4 \mathrm{k} N_{\mathrm{j}} \quad, \quad \mathrm{p}_{\mathrm{j}}=\prod_{\mathrm{j}} \hat{\mathrm{p}}=\mathrm{e}^{-H_{\mathrm{j}} / \mathrm{k} T}=\mathrm{e}^{-4 N_{\mathrm{j}}} \\
S=\sum_{\mathrm{j}} S_{\mathrm{j}}=-\mathrm{k} \ln \mathrm{p}=4 \mathrm{k} N \quad, \quad \mathrm{p}=\prod_{\mathrm{j}} \mathrm{p}_{\mathrm{j}}=\mathrm{e}^{-H / \mathrm{k} T}=\mathrm{e}^{-4 N}
\end{array}
$$

for statistically independent clusters.

According to Equations (89-91), entropy of ideal gas and photon gas are related to the number of Heisenberg-Kramers [51] or Planck [94] virtual oscillators. Due to inevitable presence of dissipations in all real processes, energy is transformed from macroscopic ordered motions to microscopic random motions known as heat thus increasing the entropy of the system by Equations (77-78). Also, by Equation (89), atomic entropy is 4 times Boltzmann universal constant $\mathrm{k}$ and hence independent of particular chemical species. Furthermore, from Equations (91) and (32), molar entropy of ideal gas is equal to universal gas constant $\tilde{S}=N^{\mathrm{o}} \mathrm{K}=R^{\mathrm{o}}$. Direct connection between entropy and heat according to its macroscopic definition in Equation (77) of Clausius or Equation (78) reveals its objective nature as opposed to its subjective interpretation in Shannon information theory [142]. For example, when liquid water evaporates at constant pressure and temperature, the relatively small number of large oscillators in liquid phase convert to large numbers of small oscillators in vapor phase thus increasing entropy $S=4 \mathrm{k} N$ while keeping atomic energy $\hat{\varepsilon}=\mathrm{k} T$ constant [33]. With oscillators acting as composite 
bosons [53, 54], like photon gas, equilibrium energy spectrum of ideal gas is given by invaraint Planck [94] distribution in Equation (5b) that is weakly dependant on the number of oscillators on account of their large number, $10^{26}$ per one kmol that is practically infinite.

As stated in Section 3, space is a compressible tachyon fluid in harmony with perceptions of Aristotle [112], Huygens [88], Newoton [99], Euler [146], Lorentz [100], Poincaré [101-103], de Broglie [3], Casimir [75], and Dirac [86] amongst others. With Casimir [75] vacuum identified as superfluid liquid photon or Bose-Einstein condensate, it is reasonable to anticipate that when heated to a critical vaporization or boiling temperature $T_{\mathrm{b}}$, space will nucleate photon gas bubbles that following Dirac [142] could be referred to as holes or anti-matter particles. Similarly, if Casimir vacuum is cooled below certain critical fusion or melting temperature $T_{\mathrm{m}}$, liquid photon is expected to undergo a second phase transition and become solidified thus forming solid-light that was defined as black hole BH [148]. Such phase transition of physical space is harmonious with finite zero point energy hence zero-point pressure of Casimir [75] vacuum according to the modified form of the van der Waals law of corresponding states [149]

$$
p_{\mathrm{rg}}=p_{\mathrm{ra}}-p_{\mathrm{rv}}=\frac{1}{\mathrm{Z}_{\mathrm{c}}}\left[\frac{\mathrm{T}_{\mathrm{r}}}{\tilde{\mathrm{v}}_{\mathrm{r}}-1 / 3}-\frac{9}{8 \tilde{\mathrm{v}}_{\mathrm{r}}^{2}}+\mathrm{Z}_{\mathrm{c}}-\frac{3}{8}\right]
$$

with predictions that are closer to experimental data as shown in Figure 8.

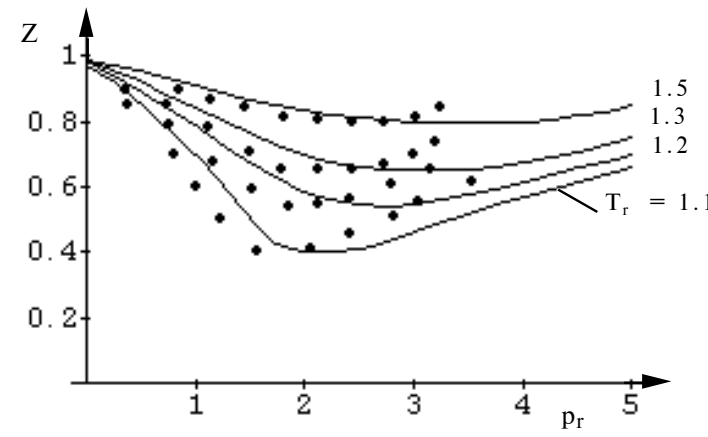

(a)

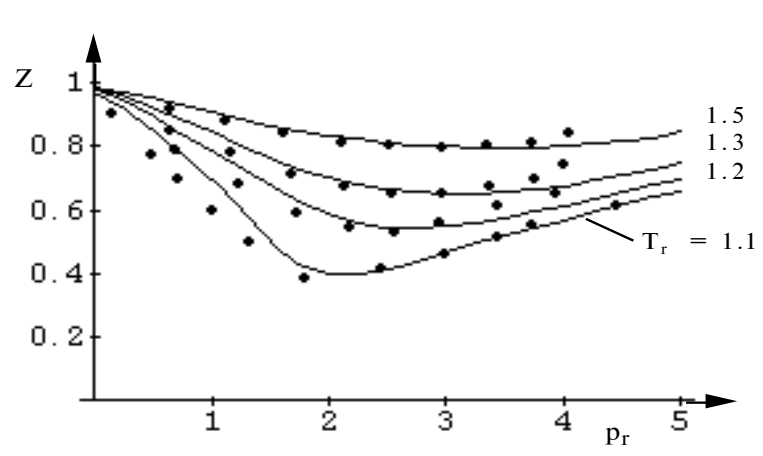

(b)

Figure 8. Comparisons between experimental data (solid lines) and the calculations (solid circles) based on (a) van der Waals (b) modified van der Waals equation of state [149]

Since $\tilde{V}_{\mathrm{r}} \rightarrow \infty$ leads to $p_{\mathrm{ra}} \rightarrow 0$ and for most substances $\mathrm{Z}_{\mathrm{c}} \leq \mathrm{Z}_{\mathrm{cVDW}}=3 / 8$, Equation (92) results in positive vacuum pressure [149]

$$
p_{\mathrm{rv}}=\frac{3 / 8}{\mathrm{Z}_{\mathrm{c}}}-1 \geq 0
$$

Also, since pressure can be viewed as volumetric potential energy density of the field, negative pressures are expected to be non-physical [34]. It is reasonable to suggest that Dirac Sea be associated with anti-matter and correspond to pressures lower than that of Casimir [75] vacuum $0 \leq p_{\mathrm{ra}} \leq p_{\mathrm{rv}}$ thus helping to avoid negatively induced cosmological constant [150].

Therefore, pressures of matter $p_{\mathrm{m}}$ and anti-matter $p_{\mathrm{am}}$ will be respectively larger and smaller than pressure $p_{\mathrm{v}}$ of Casimir vacuum [149]

$$
0=p_{\mathrm{wH}}<p_{\mathrm{am}}<p_{\mathrm{v}}<p_{\mathrm{m}}<p_{\mathrm{BH}}=\infty
$$

with white hole $p_{\mathrm{WH}}=0$ and black hole $p_{\mathrm{BH}}=\infty$ pressures identified as two singularities of the field. A schematic diagram of physical space as a compressible tachyon fluid from infinite rarefaction (white hole $\mathrm{WH}$ ) to infinite compression (black hole $\mathrm{BH}$ ) is shown in Figure 9. 


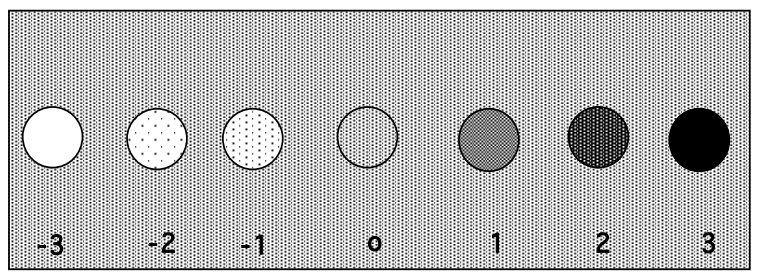

Figure 9. Different degrees of rarefaction and compression of Casimir vacuum identified as a compressible fluid. (-3) White hole $\rho_{\mathrm{wH}}=0 \quad(-2,-1)$ Anti-matter $\rho_{\mathrm{AM}}<\rho_{\mathrm{v}}(\mathbf{0})$ Casimir vacuum $\rho=\rho_{\mathrm{v}}(\mathbf{1}, \mathbf{2})$ Matter $\rho_{\mathrm{MA}}>\rho_{\mathrm{v}}$

$$
\text { Black hole } \rho_{\mathrm{BH}}=\infty \text { [37] }
$$

The temperature limits $\left(T_{\mathrm{BH}} \rightarrow O, T_{\mathrm{wH}} \rightarrow \infty\right)$ in Figure 9 correspond to formation of black hole $\mathrm{BH}$ (white hole WH) at the circumference (center) of Poincaré disk [77. 117]. Also, the model of physical space shown in Figure 9 is in harmony with previous study [34] concerning some implications of the modified form of first and second laws of thermodynamics to the important problem of black hole mechanics [151-158] emphasized by Wald [157]

"It appears that these laws of "black hole mechanics" and the laws of thermodynamics are two major pieces of a puzzle that fit together so perfectly that there can be little doubt that this "fit" is of deep significance"

In particular, rather than being related to the surface area of its horizon according to Bekenstein [153], entropy of black hole relates to its total thermal energy namely its enthalpy $H_{\mathrm{BH}}=T_{\mathrm{BH}} S_{\mathrm{BH}}=4 \mathrm{k} N_{\mathrm{BH}} T_{\mathrm{BH}}$ given in Equation (79) as $S_{\mathrm{BH}}=4 \mathrm{k} N_{\mathrm{BH}}$ in exact agreement with prediction of Major and Setter [159].

\section{Nernst, Planck, Poincaré, and the Third Law of Thermodynamics}

The third law of thermodynamics concerns the absolute minimum basis for both energy and entropy of the system. Nernst-Planck statement of the third law of thermodynamics is that both entropy and absolute temperature can reach absolute zero only through infinite number of finite increments [34, 108, 125]. Because by Equations (20) and (91) absolute temperature and entropy are respectively related to Wien wavelength and number of Heisenberg-Kramers [51] virtual oscillators, Nernst-Planck statement involving step-wise approach to zero is in harmony with quantized nature of both $T_{\beta}$ and $S_{\beta}$.

As discussed in Section 3, absolute temperature or Wien wavelength $T_{\beta}=\lambda_{w \beta}$ provides an internal "measure" of spatial extensions. It is most interestingly that in his description of hyperbolic geometry, Poincaré [117] anticipated the role of absolute thermodynamic temperature as measure or metric of physical space,

"Suppose, for example, a world enclosed in a large sphere and subject to the following laws: --The temperature is not uniform; it is greatest at the center, and gradually decreases as we move towards the circumference of the sphere, where it is absolute zero. The law of this temperature is as follows: -- If $R$ be the radius of the sphere, and $r$ the distance of the point considered from the center, the absolute temperature will be proportional to $R^{2}-r^{2}$. Further, I shall suppose that in this world all bodies have the same coefficient of dilatation, so that the linear dilatation of any body is proportional to its absolute temperature. Finally, I shall assume that a body transported form one point to another of different temperature is instantaneously in thermal equilibrium with its new environment. There is nothing in these hypotheses either contradictory or unimaginable. A moving object will become smaller and smaller at it approaches the circumference of the sphere. Let us observe, in the first place, that although from the point of view of our 
ordinary geometry this world is finite, to the inhabitants it will appear infinite. As they approach the surface of the sphere, they become colder, and at the same time smaller and smaller. The steps they take are therefore smaller and smaller, so that they can never reach the boundary of the sphere. If to us geometry is only the study of the laws according to which invariable solids move, to these imaginary beings it will be the study of the laws of motion of solids deformed by the differences of temperature alluded to."

Therefore, $T_{\beta}=\lambda_{\mathrm{w} \beta}$ is called Poincaré thermal measure of extension.

By Equation (36), one introduces the hierarchy of quantized spatial coordinate $x_{\beta}=x_{\beta}^{\prime} / \lambda_{\mathrm{w} \beta}=N_{\mathrm{x} \beta}$ at scale $\beta$ that is normalized with respect to the measure given by its temprature $T_{\beta}=\lambda_{\mathrm{w} \beta}$ thus arriving at the hierarchy [36]

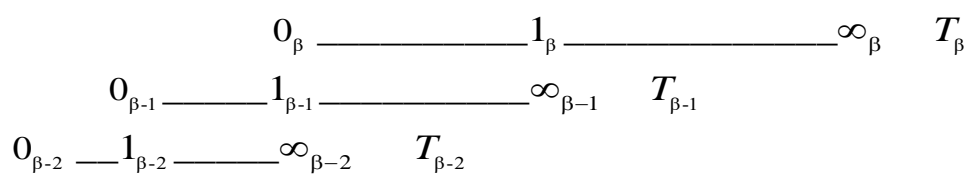

For example, for statistical fields at (ECD, EMD, EAD) scales shown in Figure 5, the cascade in Equation (95) corresponds to distributions for different atomic masses at the same temperature $T=300$ [m]. However, if one considers a constant atomic mass but varying temperatures, the hierarchy of statistical fields with different measures in Equation (95) will correspond to the cascade

$$
\begin{aligned}
T_{\beta}=0 \Leftrightarrow & T_{\beta-1}=1 \Leftrightarrow \\
T_{\beta-1}=0 \Leftrightarrow & T_{\beta-2}=\infty \\
& T_{\beta-2}=1 \Leftrightarrow T_{\beta-3}=\infty \\
& T_{\beta-2}=0 \Leftrightarrow T_{\beta-3}=1 \Leftrightarrow T_{\beta-4}=\infty
\end{aligned}
$$

Hence, to reach absolute zero temperature requires infinite number of steps, each step corresponding to a generation of statistical field with its own Nernst-Planck limits $\left(T_{\beta} \rightarrow 0_{\beta}, S_{\beta} \rightarrow 0_{\beta}\right)$ expressed as

$$
T_{\beta}=<\lambda_{\mathrm{w \beta}}^{2}>^{1 / 2} \rightarrow 0_{\beta} \quad, \quad S_{\beta}=4 N_{\beta} \mathrm{k} \rightarrow 0_{\beta}
$$

However, by Equation (95), zero of scale $\beta\left(\lambda_{\mathrm{w} \beta}=0_{\beta}, N_{\beta}=0_{\beta}\right)$ is unity $\left(\lambda_{\mathrm{w} \beta-1}=1_{\beta-1}, N_{\beta-1}=1_{\beta-1}\right)$ of scale $\beta-1$, leading to an infinite regression thus making absolute zero unattainable by finite number of steps. The stepwise (-quantized) approach of temperature, entropy, and Gibbs free energy $G_{\beta}=H_{\beta}-T_{\beta} S_{\beta}$ towards absolute zero is schematically shown in Figure 10 [34].

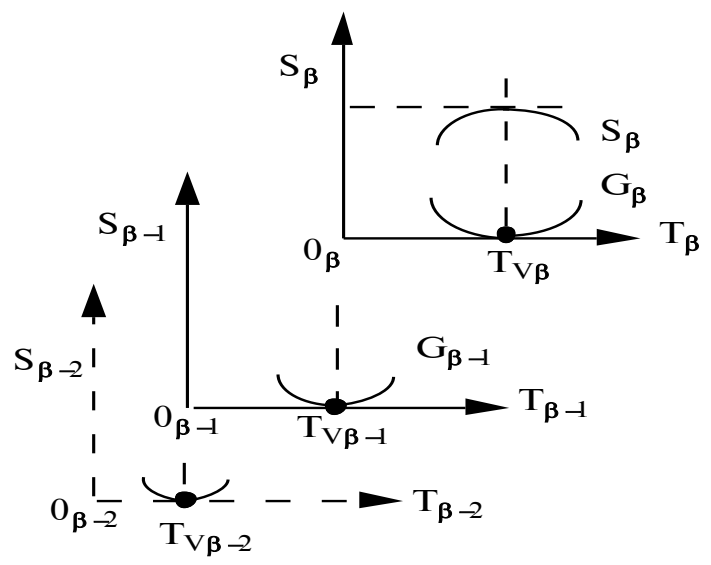

Figure 10. Hierarchy of absolute "zero" $T_{\beta} \rightarrow 0_{\beta}$ and "vacuum" $T_{\mathrm{v} \beta} \quad$ temperatures and associated entropy $S_{\beta}$ and Gibbs free energy $G_{\beta}[34]$ 
As emphasized by the quotation from Anaxagoras in Section 3, absolute zero extension hence temperature and duration hence time constitute singularities of the field and as idealized Aristotle limits in Equation (97) are never actualized. Acccording to Figure 10, each scale has a Casimir vacuum temperature that is finite and a vaccuum-vacuum temperature corresponding to the zero-measure of that scale. Also, as discussed in [36] and shown in the hierarchy of scales in Figure 10

$$
(\text { vacuum }- \text { vacuum })_{\beta}=(\text { vacuum })_{\beta-1}
$$

The quantized approach towards the limit $\left(T_{\beta} \rightarrow 0_{\beta}, S_{\beta} \rightarrow 0_{\beta}\right)$ viewed from a different perspective also represents quantized approach towards Casimir [75] zero-point energy $\left(T_{\beta} S_{\beta} \rightarrow O_{\beta}\right)$ because of the definition of enthalpy

$$
\Delta T_{\beta} \Delta S_{\beta}=T_{\mathrm{H} \beta} S_{\mathrm{H} \beta}-T_{\mathrm{L} \beta} S_{\mathrm{L} \beta}=H_{\mathrm{H} \beta}-H_{\mathrm{L} \beta}=\Delta H_{\beta}
$$

as schematically shown on $(S-T)$ diagram in Figure 11

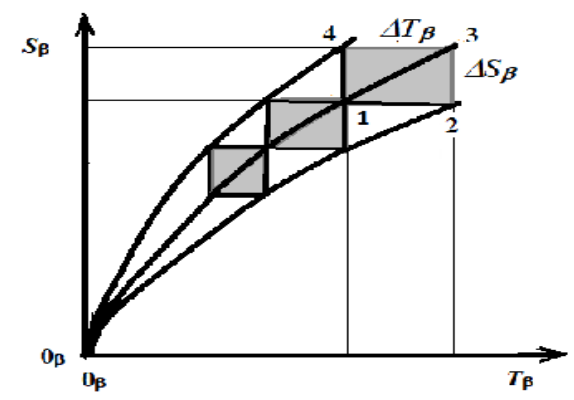

Figure 11. Stepwise asymptotic approach of entropy $S_{\beta}$ and temperature $T_{\beta}$, hence enthalpy $H_{\beta}$, towards absolute zero [34].

In reference to Carnot cycle shown in Figure 4, the net energy removed from the system at each step in Figure 11 corresponds to isentropic cooling by expansion in turbine $(3 \rightarrow 4)$ and isothermal cooling in condenser $(4 \rightarrow 1)$ hence the total area $\Delta H=\mathrm{A}_{1234}$ shown on $S$ - $T$ diagram in Figure 11 .

\section{Concluding Remarks}

A universal scale-invariant model of Boltzmann statistical mechanics and the associated four laws of generalized thermodynamics were described. Connecting Planck and Boltzmann universal constants to spatio-temporal aspects of Casimir vacuum fluctuations, Kelvin absolute temperature $T$ (degrees kelvin) was identified as Wien wavelength $T_{\beta}=\lambda_{\text {w } \beta}$ of thermal oscillations. It was shown that given temperature and atomic mass of the field $\mathbb{F}_{\beta}\left(T_{\beta}, \mathrm{m}_{\beta}\right)$ provides the background space $\mathbb{F}_{\beta}=\mathbb{S}_{\beta+1}$ required for definitions of external space and time coordinates and atomic-mass-unit of $\mathbb{F}_{\beta+1}\left(\mathrm{x}_{\beta+!}, \mathrm{t}_{\beta+!}, \mathrm{m}_{\beta+1}\right)$. The thermodynamic spacetime and Boltzmann factor are in harmony with modern concepts of deterministic dissipative quantum gravity [73]. De Pretto number 8338 and its connections to classical Joule-Mayer mechanical equivalent of heat $J_{\mathrm{c}}=4.169 \mathrm{~kJ} / \mathrm{kcal}$ and universal gas constant $R^{\mathrm{o}}=8338 \mathrm{~J} / \mathrm{kcal} . \mathrm{m}$ were identified leading to introduction of modified mechanical equivalent of heat $J=2 J_{\mathrm{c}}=8338 \mathrm{~J} / \mathrm{kcal}$. 
Helmholtz decomposition of total thermal energy into free heat $U$ and latent heat $p V$ and identification of work as Helmholtz free energy $W=F=U-T S$ resulted in modified form of the first law of thermodynamics $Q=H=U-W=U+p V$. Introducing a modified expressions for the number of photons in a given volume of Casimir vacuum, total energy under Planck energy distribution was shown to be enthalpy $H$ rather than internal energy $U$. In harmony with a modified van der Waals law of corresponding states, a model of physical space as a compressible tachyon fluid from infinite rarefaction (white hole $\mathrm{WH}$ ) to infinite compression (black hole $\mathrm{BH}$ ) was described. It is found that, rather than being related to the surface area of its horizon according to Bekenstein [153], entropy of black hole is related to its total thermal energy namely its enthalpy and given by $S_{\mathrm{BH}}=4 \mathrm{k} N_{\mathrm{BH}}$ in exact agreement with prediction of Major and Setter [159].

\section{Acknoledgments}

This research was supported by NASA Grant no. NAG3-1863 and in part supported by U.S. National Science Foundation Grant No. CTS-8820077.

\section{References}

[Ref.1] de Broglie, L. Interference and corpuscular light. Nature 1926,118, 2969, 441- 442 ; Sur la possibilité de relier les phénomènes d'Interférence et de diffraction à la théorie des quanta de lumière. C. R. Acad. Sci. Paris, 1927,183, 447448; La structure atomique de la matière et du rayonnement et la mécanique ondulatoire. 1927, 184, 273-274; Sur le rôle des ondes continues en mécanique ondulatoire. 1927, 185, 380-382.

[Ref.2] de Broglie, L. Non-Linear Wave Mechanics : A Causal Interprétation, Elsevier, New York, 1960.

[Ref.3] de Broglie, L. The Reinterpretation of Wave Mechanics. Found. Phys. 1970, 1, 5-15.

[Ref.4] Madelung, E. Quantentheorie in hydrodynamischer form. Z. Physik. 1926, 40, 332-326.

[Ref.5] Schrödinger, E. Über die Umkehrung der Naturgesetze. Sitzber Preuss Akad Wiss Phys-Math Kl, 1931,144-153.

[Ref.6] Fürth, R. Über einige beziehungen zwischen klassischer staristik und quantenmechanik. Z. Phys. 1933, 81, 143162.

[Ref.7] Bohm, D. A suggested interpretation of the quantum theory in terms of "hidden" variables. Phys. Rev. 1952, 85, 166-179.

[Ref.8] Takabayasi, T. On the foundation of quantum mechanics associated with classical pictures. Prog. Theor. Phys. $1952,8,143-182$.

[Ref.9] Bohm, D.; Vigier, J. P. Model of the causal interpretation of quantum theory in terms of a fluid with irregular fluctuations. Phys. Rev. 1954, 96, 208-217.

[Ref.10] Nelson, E. Derivation of the Schrödinger equation from Newtonian mechanics. Phys. Rev. 1966,150, 1079-1085.

[Ref.11] Nelson, E. Quantum Fluctuations; Princeton University Press, Princeton, New Jersey, 1985.

[Ref.12] de la Peña, L. New foundation of stochastic theory of quantum mechanics. J. Math. Phys. 1969,10, 1620-1630.

[Ref.13] de la Peña, L.; Cetto, A. M. Does quantum mechanics accept a stochastic support? Found. Phys. 1982,12,10171037.

[Ref.14] Barut, A. O. Schrödinger's interpretation of $\psi$ as a continuous charge distribution. Ann. der Phys. 1988, 7, 31-36.

[Ref.15] Barut, A. O.; Bracken, A. J. Zitterbewegung and the internal geometry of the electron. Phys. Rev. D 1981, 23, 2454-2463.

[Ref.16] Vigier, J. P. De Broglie waves on Dirac aether: A testable experimental assumption. Lett. Nuovo Cim. 1980, 29, 467-475; Gueret, Ph.; Vigier, J. P. De Broglie's wave particle duality in the stochastic interpretation of quantum mechanics: A testable physical assumption. Found. Phys. 1982, 12, 1057-1083; Cufaro, C.; Vigier, J. P. Dirac's aether in relativistic quantum mechanics. Found. Phys. 1983, 13, 253-286; Vigier, J. P. Derivation of inertia forces from the Einstein-de Broglie-Bohm (E.d.B.B) causal stochastic interpretation of quantum mechanics. Found. Phys. 1995, 25, 1461-1494. 
[Ref.17] Arecchi, F. T.; Harrison, R. G. Instabilities and Chaos in Quantum Optics; Springer-Verlag: Berlin, 1987.

[Ref.18] Reynolds, O. On the dynamical theory of incompressible viscous fluid and the determination of the criterion. Phil. Trans. Roy. Soc. A 1895, 186, 23-164.

[Ref.19] Enskog, D. Kinetische Theorie der Vorgange in Massig Verdunnten Gasen; by Almqvist and Wiksells Boktryckeri-A.B., Uppsala, 1917. English translation in: Brush, G. S. Kinetic Theory, Vol.1-3, Pergamon Press, New York, 1965.

[Ref.20] Taylor, G. I. Statistical theory of turbulence-Parts I-IV. Proc. Roy. Soc. A 1935,151, 421-478.

[Ref.21] Kármán, T.; Howarth, L. On the statistical theory of isotropic turbulence. Proc. Roy. Soc. A 1938,164, 192-215.

[Ref.22] Robertson, H. P. The invariant theory of isotropic turbulence. Proc. Camb. Phil. Soc. 1940, 36, 209-223.

[Ref.23] Kolmogoroff, A. N. Local structure on turbulence in incompressible fluid. C. R. Acad. Sci. U. R. S. S. 1941, 30, 301-305; Dissipation of energy in locally isotropic turbulence. C. R. Acad. Sci. U. R. S. S. 1942, 32, 19-21; A refinement of previous hypothesis concerning the local structure of turbulence in a viscous incompressible fluid at high Reynolds number. J. Fluid Mech. 1962,13, 82-85.

[Ref.24] Obukhov, A. M. On the distribution of energy in the spectrum of turbulent flow. C. R. Acad. Sci. U. R. S. S. 1941, 32, 19-22; Some specific features of atmospheric turbulence. J. Fluid Mech. 1962,13, 77-81.

[Ref.25] Chandrasekhar, S. Stochastic problems in physics and astronomy. Rev. Mod. Phys. 1943, 15, 1-89.

[Ref.26] Chandrasekhar, S. Stochastic, Statistical, and Hydrodynamic Problems in Physics and Astronomy, Selected Papers, vol.3; University of Chicago Press, Chicago, 1989, 199-206.

[Ref.27] Heisenberg, W. On the theory of statistical and isotropic turbulence. Proc. Roy. Soc. A 1948, 195, 402-406; Zur Statistischen Theorie der Turbulenz. Z. Phys. 1948, 124, 628-657.

[Ref.28] Batchelor, G. K. The Theory of Homogeneous Turbulence; Cambridge University Press, Cambridge, 1953.

[Ref.29] Landau, L. D.; Lifshitz, E. M. Fluid Dynamics; Pergamon Press, New York, 1959.

[Ref.30] Tennekes, H.; Lumley, J. L. A First Course in Turbulence; MIT Press, 1972.

[Ref.31] Sohrab, S. H. Invariant forms of conservation equations and some examples of their exact solutions. J. Energy Resources Technol. 2014, 136, 1-9.

[Ref.32] Sohrab, S. H. Solutions of modified equation of motion for laminar flow across (within) rigid (liquid) sphere and cylinder and resolution of Stokes paradox. AIP Conference Proceedings 1896, 130004, 2017.

[Ref.33] Sohrab, S. H. Some implications of a scale invariant model of statistical mechanics to classical and relativistic thermodynamics. Int. J. Thermo. 2014, 17, 4, 233-248.

[Ref.34] Sohrab, S. H. On a scale-invariant model of statistical mechanics and the laws of thermodynamics. J. Energy Resources and Technol. 2016, 138, 3, 1-12.

[Ref.35] Sohrab, S. H. Quantum theory of fields from Planck to cosmic scales. WSEAS Trans. Math. 2010, 9, 734-756.

[Ref.36] Sohrab, S. H. On a scale invariant model of statistical mechanics, kinetic theory of ideal gas, and Riemann hypothesis. Int. J. Mod. Communication Tech. \& Research. 2015, 3, 6, 7-37.

[Ref.37] Sohrab, S. H. Connecting Bernoulli and Schrödinger equations and its impact on quantum mechanics wave function and entanglement problems. Skiadas C. H.; Dimotikalis, Y., eds.; 13th Chaotic Modeling and Simulation International Conference, Springer Proceedings in Complexity, 2021.

[Ref.38] Ván. P., Weakly nonlocal irreversible thermodynamics. Ann. Phys. (Leipzig) 2003, 12, 3, 146-173.

[Ref.39] El-Nabulsi, R.A. Nonlocal approach to nonequilibrium thermodynamics and nonlocal heat diffusion processes, Continuum Mechanics and Thermodynamics 2018,30, 889-915.

[Ref.40] El-Nabulsi, R.A. Nonlocal Thermodynamics Properties of Position-Dependent Mass Particle in Magnetic and Aharonov-Bohm Flux Fields, Few-Body Syst. 2020, 61:37, 1-12.

[Ref.41] El-Nabulsi, R.A. Non-standard power-law Lagrangians in classical and quantum dynamics. Appl. Math. Lett. 2015, 43, 120-127.

[Ref.42] Barretta, R.; Čanađija, M.; Marotti de Sciarra, F. Nonlocal integral thermoelasticity: A thermodynamic framework for functionally graded beams, Composite Structures 2019, 225, 111104.

[Ref.43] de Groot, R. S.; Mazur, P. Nonequilibrium Thermodynamics; North-Holland, Amsterdam, 1962.

[Ref.44] Schlichting, H. Boundary-Layer Theory; McGraw Hill, New York, 1968. 
[Ref.45] Williams, F. A. Combustion Theory; Addison Wesley, New York, 1985.

[Ref.46] Hirschfelder, J. O.; Curtiss, C. F.; Bird, R. B. Molecular Theory of Gases and Liquids; John Wiley: New York, 1954.

[Ref.47] Chapman, S.; Cowling, T. G. The Mathematical Theory of Non-uniform Gases; Cambridge University Press, Cambridge, 1953.

[Ref.48] 't Hooft, G. How does God play dice? (Pre-) determinism at the Planck scale, arXiv:hep-th/0104219v1 25, April, 2001.

[Ref.49] Born, M. Zur Quantenmechanik der Stoßvorgänge. Z. Physik. 1926, 37, 863.

[Ref.50] Bokulich, A. Reexamining the Quantum-Classical Relation; Cambridge University Press, New York, 2008.

[Ref.51] van der Waerden, B. L. Towards Quantum Mechanics, in: Sources of Quantum Mechanics, van der Waerden, B. L. Ed.; Dover: New York, 1967, pp. 1-59.

[Ref.52] Poincaré, H. Sur le problèm des trois corps et les équations de la dynamique. Mémoire courroné du prix de S. M. le roi Oscar II de Suède. Acta Math. 1890, 13, A3-A270.

[Ref.53] Laloe, F.; Freed, J. H. The effects of spin in gases. Sci. American 1988, 258, 94-101.

[Ref.54] Sohrab, S. H. The physical foundation of a grand unified statistical theory of fields and the invariant Schrödinger equation. WSEAS Transactions on Circuits and Systems 2004, 3, 4, 1017-1025.

[Ref.55] Sonntag, R. E.; van Wylen, G. J. Fundamentals of Statistical Thermodynamics; John Wiley, New York, 1966.

[Ref.56] Kardar, M. Statistical Physics of Particles; Cambridge University Press, New York, 2007.

[Ref.57] Krout, K. A.; Sohrab, S. H. On the speed of sound. Int. J. Thermodynamics 2016, 19, 1, 29-34.

[Ref.58] Poincaré, H. The present and the future of mathematical physics. Address delivered before the Section of Applied Mathematics of the International Congress of Arts and Science, St. Louis, September 24, 1904; Bull. Amer. Math. Soc. 2000, 37, 1, 25-38.

[Ref.59] Gouy, M. Note sur le mouvement Brownien. J. de Phys. 1888, 7, 561-564; Sur le mouvement Brownien. C. R. Acad. Sci. Paris 1889, 109, 102-105; Le mouvement Brownien et les mouvement moléculaires. Rev. Gén. Sci. 1895, 1, 6, 1-7.

[Ref.60] Bachelier, L. Théorie mathématique du jeux. Ann. Sci. Ecole Normale Supérieure 1900, 21-86.

[Ref.61] Cercignani, C. Ludwig Boltzmann, The Man Who Trusted Atoms; Oxford University Press, Oxford, 1998.

[Ref.62] Sutherland, W. A. Causes of osmotic pressure and of the simplicity of the laws of dilute solutions. Philos. Mag. S.5, 44, 1897, 52-55; Ionization, ionic velocities, and atomic sizes. Philos. Mag. 1902, 4, 625-645; A dynamic theory for non-electrolytes and the molecular mass of albumin. Philos. Mag. 1905, 9, 781-785.

[Ref.63] Smoluchowski, M. Polish Men of Science; Ingarden, R. S. (Ed.), Polish Science Publishers, Warszawa, 1986.

[Ref.64] Einstein, A. Investigations on the theory of Brownian movement. Fürth, R., Ed.; Dover Publications: New York, 1956.

[Ref.65] Perrin, J. M. Brownian Movement and Molecular Reality; Taylor and Francis: London, 1910.

[Ref.66] Uhlenbeck, G. E.; Ornstein, L. S. On the theory of the Brownian motion. Phys. Rev. 1930, 36, 823-841.

[Ref.67] Chen, W. M.; Uhlenbeck, G. E. On the theory of the Brownian motion II. Rev. Mod. Phys. 1945, 17, 2-3, 323342.

[Ref.68] Füchs, N. A. The Mechanics of Aerosols; Dover: New York, 1964.

[Ref.69] Nelson, E. Dynamical Theory of Brownian Motion; Princeton University Press: Princeton, 1967.

[Ref.70] Duplantier,B. Brownian motions, diverse and undulating. In: Einstein 1905-2005, Poincaré Seminar 2005. T. Damour, O. Darrigol, Duplantier, B.; and Rivasseau, V., Eds.; Birkhäuser, 2006, pp. 201-293.

[Ref.71] van der Waerden, B. L. Sources of Quantum Mechanics, B. L. van der Waerden, ed.; Dover: New York, 1967.

[Ref.72] 't Hooft, G. The Cellular Automaton Interpretation of Quantum Mechanics; Springer: Cham, Switzerland, 2016.

[Ref.73] 't Hooft, G. Quantum gravity as a dissipative deterministic system. Class. Quantum Grav. 1999, 16, 3263-3279.

[Ref.74] Heisenberg, W. The Physical Principles of Quantum Theory; Dover: New York, 1949.

[Ref.75] Casimir, H. B. G. On the attraction between two perfectly conducting plates. Proc. K. Ned. Akad. Wet. 1948, 51, 793-795. 
[Ref.76] Connes, A. On the Fine Structure of Spacetime. In: On Space and Time, Shahn M., ed.; Cambridge University Press, 2008, pp. 196-237.

[Ref.77] Sohrab, S. H. An invariant model of Boltzmann statistical mechanics and some of its implications to thermodynamics and quantum nature of space and time. WSEAS Tran. Appl. Theo. Mech. 2018, 13, 199-212.

[Ref.78] Rovelli, C. Reality is Not What it Seems; Riverhead Books, New York, 2017, p. 183.

[Ref.79] Rovelli, C.; Smolin, L. Loop space representation of quantum general relativity. Nucl. Phys. B 1990, 331, 80152.

[Ref.80] Ashtekar, A. The winding road to quantum gravity. Current Science 2005, 89, 12, 2064-2074.

[Ref.81] DeWitt, S. B. Quantum theory of gravity. I. The canonical theory. Phys. Rev. 1967, 160, 1113-114.

[Ref.82] Wheeler, J. A. Super space and the Nature of Quantum Geometro-dynamics. In: Battelle Rencontres, DeWitt C. M., and Wheeler J. A., eds.; Benjamin: New York 1968, 242-307.

[Ref.83] Sohrab, S. H. Some thermodynamic considerations on physical and quantum nature of space and time. ECOS 2000 Proceedings, University of Twente, Enschede, Nederland, July 5-7, 2000; WSEAS Trans. Math. 2004, 3, 4, 764772.

[Ref.84] Sohrab, S. H. Invariant model of statistical mechanics, quantum mechanics, and physical nature of space and time. 8th CHAOS Conference Proceedings, 26-29 May 2015, 769-801, Henri Poincaré Institute, Paris, France.

[Ref.85] Planck, M. Where Is Science Going; Ox Bow Press: Woodbridge, Connecticut, 1981.

[Ref.86] Dirac, P. A. M. Is there an aether?; Nature 1951, 168, 906.

[Ref.87] El-Nabulsi, R.A., Some implications of three generalized uncertainty principles in statistical mechanics of an ideal gas, Few-Body Syst 2020, 61:37, 1-12.

[Ref.88] Huygens, C. Treatise on Light; Dover, 1912, p. 14.

[Ref.89] Lorentz, H. A. Selected Works of H. A. Lorentz; Neressian, N. J., and Cohen, F. H. , eds.; Palm Publications, Nieuwerkerk, 1987.

[Ref.90] Verhulst, F. Henri Poincaré, Impatient Genius; Springer, 2012, p. 64.

[Ref.91] Sohrab, S. H. Scale invariant model of statistical mechanics and quantum nature of space, time, and dimension. Chaotic Modeling and Simulation (CMSIM) 2016, 3, 231-245.

[Ref.92] Darrigol, O. The mystery of the Einstein Poincare' connection. Isis 2004, 95, 614-626.

[Ref.93] Galison, P. Einstein's Clocks, Poincaré's Maps; W. W. Norton \& Company, New York, 2003.

[Ref.94] Planck, M. On the law of the energy distribution in the normal spectrum. Ann. der Phys. 1901, 4, 553-558.

[Ref.95] Darrigol, O. Statistics and combinatorics in early quantum theory. Historical Studies in the Physical and Biological Sciences, 1988, 19, 1, 17-80.

[Ref.96] Darrigol, O. Statistics and combinatorics in early quantum theory, II: Early symptoms of indistinguishability and holism. Historical Studies in the Physical and Biological Sciences 1991, 21, 2, 237-298.

[Ref.97] de Broglie, L. Matter and Light-The New Physics; Dover: New York, 1937.

[Ref.98] Lochak, G. The evolution of the ideas of Louis de Broglie on the interpretation of wave mechanics. In: Quantum, Space and Time-The Quest Continues, Asim O. Barut, Alwyn van der Merwe, and Jean-Pierre Vigier, eds.; Cambridge University Press, 1984, pp. 11-33.

[Ref.99] Newton, I. Optics; Dover: New York, 1952.

[Ref.100] Lorentz, H. A. Electromagnetic phenomena in a system moving with any velocity less than that of light. in: The Principles of Relativity; Dover, 1952.

[Ref.101] Poincaré, H. La théorie de Lorentz et le principe de réaction. Arch. Neerland. 1900, 5, 252-278; http://www.physicsinsights.org/poincare-1900.pdf.

[Ref.102] Poincaré, H. Sur la dynamique de l'electron. C. R. Acad. Sci. Paris 1905, 140, 1504-1508.

[Ref.103] Poincaré, H. Sur la dynamique de l'electron. Rend. Circ. Mat. Palermo 1906, 21, 9-175.

[Ref.104] de Pretto, O. Ipotesi dell'etere nella vita dell'universo. Reale, Inst. Veneto di Scienze, Lettere ed Arti 1904, 63, 439-500. 
[Ref.105] Hasenöhrl, F. Zur theorie der strahlung in bewegten körpern. Ann. der Physik 1904, 15, 344-370; Zur theorie der strahlung in bewegten körpern. Ann. der Phys. 1905, 16, 589-592.

[Ref.106] Einstein, A. Zur Elecrodynamik bewegter Körpern. Ann. der Phys. (Leipzig) 1905, 17, 891-921.

[Ref.107] Long, C. A.; Sohrab, S. H. The power of two, speed of light, force and energy and the universal gas constant. In: Recent Advances on Applied Mathematics, Long, C. A.; Sohrab, S. H.; Bognar, G.; Perlovsky, L., Eds.; WSEAS Press, 2008, 434-442.

[Ref.108] Pauli, W. Pauli Lectures on Physics; MIT Press, 1973, p. 14, volume 3.

[Ref.109] 't Hooft, G.; Vandoren, S. Time in Powers of Ten; World Scientific: Singapore, 2014.

[Ref.110] Minkowski, H. Space and time. in: Theory of Relativity; Dover: New York, 1952, p. 75.

[Ref.111] Rovelli, C. Statistical mechanics of gravity and the thermodynamical origin of time. Class. Quantum Grav. 1993, 10, 1549-1566.

[Ref.112] Aristotle, In: Time, Westphal J.; Levenson, C., Eds.; Hackett Publishing Company: Indianapolis, Indiana, 1993.

[Ref.113] Augustine, St. In: Time; Westphal J. and Levenson, C., Eds.; Hackett Publishing Company: Indianapolis, Indiana, 1993.

[Ref.114] Penzias, A. A.; Wilson, R. W. A measurement of excess antenna temperature at 4080 Mc/s. APJ. 1965, 142, 419-421.

[Ref.115] Bell, J. L. The Continuous and the Infinitesimal in Mathematics and Philosophy; Polimetrica: Milano, Italy, 2006.

[Ref.116] 't Hooft, G. In Search of the Ultimate Building Blocks; Cambridge University press, 1998, p. 125.

[Ref.117] Poincaré, H. The Value of Science; Dover: New York, 1958, p. 142.

[Ref.118] Planck, M. The Theory of Heat Radiation; Dover: New York, 1959.

[Ref.119] Lemaître, G. H. J. É. A homogeneous universe of constant mass and growing radius accounting for the radial velocity of extragalactic nebulae. Monthly Notices of the Roy. Astr. Soc. Xci. 1931, 483-490 ; PNAS 1934, 20, 12-17.

[Ref.120] Everett, H. III. Relative state formulation of quantum mechanics. Rev. Mod. Phys. 1957, $29,454$.

[Ref.121] Guth, H. A.; Tye, S.-H. H. Phase transitions and magnetic monopole production in the very early universe. Phys. Rev. Lett. 1980, 44, 10, 631-634.

[Ref.122] Guth, H. A. Inflationary universe: A possible solution to the horizon and flatness problems. Phys. Rev. D 23, 2, 1981, 347-356.

[Ref.123] Albrecht, A.; Steinhardt, J. P. Cosmology for grand unified theories with radiatively induced symmetry breaking. Phys. Rev. Lett. 1982, 48, 17, 1220-1223.

[Ref.124] Linde, A. D. E. A new inflationary universe scenario: A possible solution of the horizon, flatness, homogeneity, isotropy and primordial monopole problems. Phys. Lett. B, 1982 108, 694; 1988, 389-393.

[Ref.125] Sommerfeld, A. Thermodynamics and Statistical Mechanics; Academic Press: New York, 1956.

[Ref.126] Warner, R. E. Statement of the first law of thermodynamics. Amer. J. Phys. 1961, 29, 124.

[Ref.127] Helmholtz, H. Über der Ehaltung der Kraft, Eine Physikalische Abhandlung. G. Reiner, Berlin, 1947. English translation in: G. S. Brush, Kinetic Theory, Vol.1-3; Pergamon Press: New York, 1965.

[Ref.128] Clausius, R. Über die Art der Bewegung, welche wir Wärme nennen. Annalen der Phyzik. 1957,100, 353-80. Philosophical Magazine 1957,14,108-127.

[Ref.129] Clausius, R. Ueber einen auf die Wärme anwendbaren mechanischen Satz. Sitzungsberichte der Niedderrheinischen Gesellschaft, Bonn, 1870,114-119.

[Ref.130] Sohrab, S. H. Invariant laws of thermodynamics and validity of Hasenöhrl mass-energy equivalence formula $\mathrm{m}$ $=(4 / 3) E / c^{2}$ at photonic, electrodynamic, and cosmic scales. Bull. Amer. Phys. Soc. 2017, 62, 1, K6.

[Ref.131] Turner, M. S. Dark matter in the universe, Physica Scripta 1991, T36, 167.

[Ref.132] Krauss, L. M.; Turner, M. S. The cosmological constant is back. 1995, 27, 11, 1137-1144.

[Ref.133] Turner, M. S. The case of $\Omega_{\mathrm{M}}=0.33 \pm 0.035$. ApJ. 2002,576, 2 .

[Ref.134] Dai, Z. G.; Liang, E. W.; Xu, D. Constraining $\Omega_{\mathrm{M}}$ and dark energy with gamma-ray bursts. ApJ. 2004, 612, L101-L104. 
[Ref.135] Polarski, D. Dark energy: Current issues. Ann. der Phys. 2006,19, 219-229.

[Ref.136] Riess, A. G.; et al., Observational evidence from supernovae for an accelerating universe and a cosmological constant. AJ. 1998,116, 1009.

[Ref.137] Riess, A. G.; et al. Type Ia supernova discoveries at $z>1$ from the Hubble Space Telescope: Evidence for past deceleration and constraints on dark energy evolution. ApJ. 2004, 607, 665.

[Ref.138] Schmidt, B. G.; et al. The high-Z supernovae search: Measuring cosmic deceleration and global curvature using type Ia supernovae. ApJ. 1998, 507, 46.

[Ref.139] Perlmutter, S.; et al., Measurements of $\Omega$ and $\Lambda$ from 42 high-redshift supernovae. ApJ. 1999, 517, 565.

[Ref.140] Einstein, A. Ist die Trägheit eines Körpers von seinem Energieinhalt abhängig? Ann. der Phys. 1905, 18, 639641.

[Ref.141] Pauli, W. Theory of Relativity; 135, Dover: New York, 1958.

[Ref.142] Sohrab, S. H. Boltzmann entropy of thermodynamics versus Shannon entropy of information theory. Int. J. Mech. 2014, 8, 73-84.

[Ref.143] Boltzmann, L. Lectures on Gas Theory; Dover: New York, 1964.

[Ref.144] Riek, R. A derivation of a microscopic entropy and time irreversibility from the discreteness of time. Entropy 2014, 16, 6, 3149-3172.

[Ref.145] Boltzmann, L. Weitere Studien uber das Warmegleichgewicht unter Gasmoleculen. Sitzungsberichte Akad.Wiss., Vienna, Part II, 1872, 66, 275-370. English translation in: Brush, G. S. Kinetic Theory, Vol.1-3, Pergamon Press: New York, 1965, 88-175.

[Ref.146] Euler, L. Réflexions sur l'espace et le temps. Histoire de l'Academie Royale des sciences et belles lettres 1748, 4, 324-333.

[Ref.147] Dirac, A. M. P. Directions in Physics; John Wiley: New York, 1978.

[Ref.148] Sohrab, S. H. Some implications of a scale-invariant model of statistical mechanics to classical and black hole thermodynamics. Bull. Amer. Phys. Soc. 2017, 61, 1, M15.

[Ref.149] Sohrab, S. H. Modified van der Waals equation of state. WSEAS Transactions on Biology and Biomedicine 2004, 1, 4, 422-424.

[Ref.150] El-Nabulsi, R.A. Phase transitions in the early universe with negatively induced supergravity cosmological constant, Chinese Phys. Lett. 2006, 23:5, 1124.

[Ref.151] Christodoulou, D. Reversible and irreversible transformations in black hole physics. Phys. Rev. Let. 25, 22, 1970, 1596-1597. Math. Phys. 1973, 31, 161-170.

[Ref.152] Bardeen, J. M.; Carter, B.; Hawking, S. W. The four laws of black hole mechanics. Commun. Math. Phys. 1973, 31, 161-170.

[Ref.153] Bekenstein, J. D. Black holes and entropy. Phys. Rev. D 1973, 7, 2333-2346.

[Ref.154] Hawking, S. W. Black holes and thermodynamics. Phys. Rev. D 1976, 13, 191-197.

[Ref.155] Hawking, S. W. Black hole explosions. Nature 1974, 248, 30-31.

[Ref.156] 't Hooft, G. On the quantum structure of a black hole. Nucl. Phys. B 1985, 256, 727-745.

[Ref.157] Wald, R. M. Black Holes and Thermodynamics; in: Black Holes and Relativistic Stars; Wald R. M., ed.; University of Chicago Press: Chicago, 1998.

[Ref.158] Grumiller, R. M.; McNees, R.; Salzer, J. Black hole thermodynamics: The first half century, in: Quantum Aspects of Black Holes; Fundamentals of Theoretical Physics 178, X. Calmet, ed.; Springer: Switzerland, 2015.

[Ref.159] Major, S. A.; Setter, K. L. Gravitational statistical mechanics: A model. Class. Quant. Grav. 2001, 18, 51255142. 